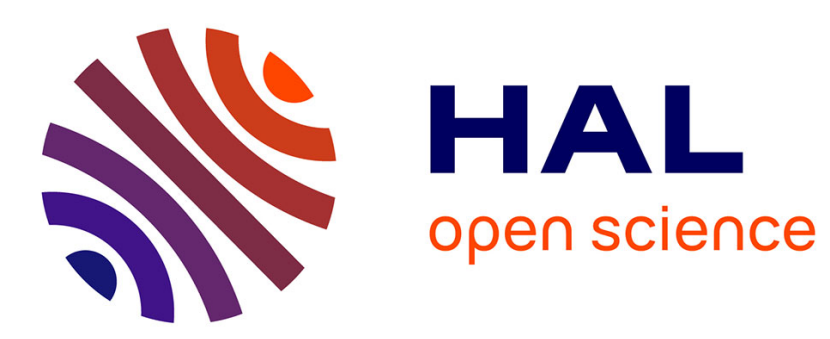

\title{
La valeur psychologique du temps: Une synthèse de la littérature
}

\author{
Hubert de La Bruslerie, Florent Pratlong
}

\section{To cite this version:}

Hubert de La Bruslerie, Florent Pratlong. La valeur psychologique du temps: Une synthèse de la littérature. Actualite Economique, 2012, 88 (3), pp.1-39. halshs-00636357

\section{HAL Id: halshs-00636357 https://shs.hal.science/halshs-00636357}

Submitted on 27 Oct 2011

HAL is a multi-disciplinary open access archive for the deposit and dissemination of scientific research documents, whether they are published or not. The documents may come from teaching and research institutions in France or abroad, or from public or private research centers.
L'archive ouverte pluridisciplinaire HAL, est destinée au dépôt et à la diffusion de documents scientifiques de niveau recherche, publiés ou non, émanant des établissements d'enseignement et de recherche français ou étrangers, des laboratoires publics ou privés. 


\title{
La valeur psychologique du temps : Une synthèse de la littérature
}

Hubert de La Bruslerie

Professeur, Université Paris Dauphine

DRM Finance

Florent Pratlong

Maître de Conférences, Université Paris I

PRISM Sorbonne

\begin{abstract}
Résumé. - Cet article procède à une synthèse de la littérature sur la question de la valeur psychologique du temps. Il discute des différents déterminants du taux d'actualisation et la possibilité d'avoir une fonction d'escompte de type hyperbolique. L'approche historique permet de retracer les origines du concept de valeur psychologique du temps. Ce concept est ensuite rediscuter dans le cadre de la théorie des perspectives, en considérant que l'utilité ressentie de l'agent vient modifier la structure de leur valeur psychologique du temps. L'analyse des résultats empiriques et des méthodologies d'identification permet de mettre en évidence que motifs individuels et psychologiques conditionnent le caractère intertemporel des choix.
\end{abstract}

Mot clés. - Taux d'actualisation, choix intertemporel, actualisation hyperbolique, préférence pour le présent

Classification JEL. : D84, D90, E21, H43

\section{The pyschological value of time: a survey of the litterature}

Summary. - This article provides an overview of the literature on the psychological value of time. It discusses various determinants of the discount rate and the possibility of hyperbolic discounting. The historical approach helps us to trace the origins of the concept of psychological value of time. This concept is then discussed again within the framework of the prospect theory, considering that perceived utility of the agent alters the structure of their psychological value of time. The analysis of empirical findings and methodologies of identification can demonstrate that individual and psychological motives condition the nature of intertemporal choice.

Keywords. - Time discounting, intertemporal choice, preference, decision making. 


\section{Introduction}

Le temps est à la fois une ressource économique, une dimension du choix et un lieu d'expression de l'utilité. Pour Kahneman et Riis, "Le temps est l'ultime ressource finie de la vie, trouver comment l'utiliser au mieux est un vrai objectif à la fois au niveau individuel et au niveau des choix publics qui sont concernés par le bien-être humain ${ }^{1}$. La plupart des choix impliquent un arbitrage benthamien entre "plaisirs et peines » évalué en termes de bénéfices et de coûts intervenant à différentes dates. Ce processus d'évaluation des bénéfices et des coûts fait référence à la dimension de l'utilité (Buchanan, 1969). Il s'y ajoute une dimension temporelle avec une analyse des choix multiples entre plusieurs périodes. L'aversion au risque et le taux d'impatience, encore appelé taux d'escompte ou taux d'intérêt psychologique, apparaissent être les éléments essentiels guidant le comportement des agents. Le temps lieu d'expression de l'utilité signifie que l'utilité qui arrive est datée. Elle s'insère dans un lieu de cadrage: le passé et le futur de l'individu. Cette mise en perspective temporelle voit son importance soulignée par les travaux des psychologues cogniticiens tels Kahneman et Tversky, qui introduisent une différence entre sur l'utilité ressentie et expérimentée opposé à l'utilité décisionnelle.

Parmi les notions nécessaires à l'évaluation intertemporelle des alternatives, il faut toutefois distinguer les deux concepts liés d'escompte du temps et de préférence temporelle. La préférence intertemporelle est une hypothèse de comportement. Elle se réfère à «une préférence pour l'utilité immédiate par rapport à une utilité future $»^{2}$. C'est parce qu'il existe une préférence temporelle que se pose au moment d'un choix économique la nécessité d'une évaluation psychologique du temps. L'escompte du temps ou taux d'impatience conduit à analyser «toutes les raisons pour lesquelles les conséquences futures sont moins importantes incluant les facteurs qui diminuent l'utilité anticipée d'une future conséquence (comme l'incertitude ou le changement des préférences) ${ }^{3}$. Cette hypothèse dite d'impatience ou de taux d'actualisation positif est parfois contestée. Elle est communément acceptée à la fois par les économistes dans la théorie des choix (Von Mises, 1949), par les psychologues cognitivistes (O'Donoghue et Rabin, 1999, Thaler, 1981) ou encore par les analystes du comportement du consommateur (Zauberman, 2003).

La théorie sous-jacente à l'analyse des choix intertemporels est fondée plus particulièrement sur l'hypothèse d'impatience ou de préférence pour l'immédiat (Von Mises, 1949) qui souligne que les plupart des agents préfèrent une utilité (bénéfice) présente à une utilité (bénéfice) future de même montant. L'analyse des choix intertemporels n'est cependant pas indépendante de la combinaison d'effets de contexte, de cheminement temporel, de mortalité et d'impatience plus ou moins grande. Ces éléments sont propres à chacun. La théorie standard de l'utilité escomptée utilise un taux d'escompte du temps stable, qui s'apparente à un prix psychologique unique du temps. Toutefois, l'inadéquation entre les observations comportementales et les prédictions théoriques standards conduit à introduire l'idée d'une structure par terme des prix psychologiques du temps vraisemblablement décroissante. Cette hypothèse permet d'enrichir la représentation standard de la modélisation statique d'une structure temporelle constante des préférences et l'analyse des choix intertemporels.

\footnotetext{
${ }^{1}$ Cf. art. cit [2005, p. 294]

${ }^{2}$ Cf. Frederick et al. [2002, p. 351]

${ }^{3}$ Cf. Frederick et al. [2002, p. 351]
} 
Cet article introduit, dans le cadre d'une rationalité économique des comportements individuels, la notion de structure par terme du prix psychologiques du temps. La valeur du temps subjective à chaque agent découle directement d'une perception individuelle des choix entre le «moi » présent et les «mois » futurs. Cette représentation nécessite notamment de prendre en compte les caractéristiques individuelles de chaque agent et plus particulièrement d'intégrer la possibilité de révision des choix. L'objectif de cet article vise à établir une synthèse de la littérature relative à la valeur psychologique du temps et voir comment ce concept a évolué suite aux apports nouveaux de la théorie psychologique expérimentaliste «prospect theory » au sens de Kahneman et Tversky. Le concept de valeur psychologique du temps est en effet repensé dans cette synthèse, au-delà des contributions de Frederick et al. [2002] ainsi que celle Soman et al. [2005], en considérant l'apport de la rationalité élargie pour expliquer les incohérences temporelles des choix de l'agent. La notion d'utilité ressentie est notamment mobilisée à travers l'intégration du cadrage de vie «framing » apporté par la psychologie cognitive. Dans l'analyse des choix intertemporels, il est ainsi important d'insister sur la notion de probabilité de survie qui vient affecter la valeur subjective du temps.

Cet article est organisé de la façon suivante. La section 1 présente le cheminement historique et théorique qui a conduit à la notion de structure par terme des préférences intertemporelles. La section 2 s'interroge sur la rationalité économique découlant de la maximisation standard de l'utilité intertemporelle. L'hypothèse d'une structure décroissante des prix psychologiques du temps alors est analysée. Elle pose les bases conceptuelles d'une reformulation des fondements théoriques pour introduire la notion de valeur psychologique du temps. La section 3 présente les résultats des travaux empiriques qui ont évalué indirectement ou par des enquêtes comportementales le prix psychologique du temps.

\section{L'évaluation intertemporelle des préférences}

\subsection{L'attitude face au temps dans la littérature}

L'importance du caractère intertemporel des choix a été soulignée dès les premiers travaux de l'analyse économique. Dans la lignée d'Adam Smith, s'interrogeant sur les conditions de la richesse des nations, Rae [1834] ouvre sur une perspective plus microéconomique en soulignant l'importance de la dimension temporelle des choix individuel de l'agent dans son désir d'accumulation de richesses. A ce titre, Rae identifie l'existence d'une préférence temporelle pour le présent de telle façon que «la présence de l'objet immédiat du désir dans l'esprit (du décideur) donne une vision très vivante des plaisirs que procure leur possession instantanée $»^{4}$. Cette vision des choix économiques humains fait ainsi une double référence à dimension d'utilité et à celle de préférence temporelle reposant sur les bases de caractéristiques individuelles. Bohm-Bawerk [1889] insiste aussi sur l'importance d'une dimension individuelle des choix intertemporels pour justifier une préférence pour la jouissance immédiate et une aversion au risque car "il se peut que nous possédons une capacité inadéquate d'imaginer et de se projeter, ou que nous ne souhaitons pas faire aucun effort nécessaire, mais à chaque occasion nous percevons une représentation plus ou moins incomplète de notre futur désir $»^{5}$. Les arbitrages intertemporels des agents font donc intervenir un taux de préférence temporelle pour le présent.

\footnotetext{
${ }^{4}$ cité par Frederick et al. [2002, p. 353]

${ }^{5}$ Cf. Bohm-Bawerk [1889], p. 268-269
} 
C'est bien plus tard que Fisher [1930] propose une formalisation microéconomique des choix intertemporels qui étudie les choix d'allocation des ressources sur plusieurs périodes. Cet arbitrage intertemporel conduit à définir le taux de préférence temporel de l'agent lorsque celui-ci est indifférent entre substituer de la consommation présente et future. Cette première représentation formelle du processus d'actualisation met ainsi en évidence l'existence d'une valeur psychologique du temps. Ce taux de préférence temporelle pour le présent, encore appelé le taux d'impatience par Fisher [1930], est fondé sur la mise en perspective de deux taux différents : d'une part un taux d'escompte objectif et collectif, $r$, correspondant au prix social du temps sur un marché, le taux d'intérêt du marché, et d'autre part un coefficient d'actualisation du futur subjectif, $\delta$, propre à chaque individu qui exprime sa préférence temporelle. La détermination de ce paramètre $\delta$ nécessite toutefois une considération de la temporalité de l'ensemble des choix de l'individu ainsi que du rapport qu'entretiennent entre eux son «moi » économique présent et ses «mois » économiques futurs. Fisher [1930] raisonne sur la comparaison de deux périodes du temps. Il met en évidence un taux marginal de substitution intertemporel. Sa temporalité est donc finie. Elle n'exclue pas à priori des taux d'actualisation différents pour chaque couple de dates quelconque.

La vision de la rationalité temporelle introduite par Von Mises [1949] apparaît singulièrement moderne pour comprendre l'évaluation intertemporelle des préférences. Selon lui, il n'est pas permis d'introduire «une différence entre l'action rationnelle et l'action prétendument irrationnelle sur la base d'une comparaison de l'action réelle avec les brouillons et les plans antérieurs en vue d'actions futures. (...) Les plans d'hier ne nous fournissent pas une norme plus objective et moins arbitraire pour l'évaluation de l'action réelle d'aujourd'hui que les autres idées et normes $»^{6}$. Les choix passés sont dictés par une information et une logique autre, qui n'est pas pertinente pour décider de l'action aujourd'hui. Entre les deux, il y a eu un fait nouveau qui est la réalisation des choix d'hier. La cohérence (consistency) temporelle parfaite ex post des choix n'est pas une exigence de rationalité dans l'action. Von Mises [1949] ajoute encore que «constance et rationalité sont des notions différentes. Si les évaluations ont changé, une fidélité sans faille, par simple souci de constance, aux principes d'action qu'on a un jour épousés ne serait pas rationnelle » ${ }^{7}$

Le temps apparaît alors comme une catégorie spécifique dans la décision. «L'action ne peut influencer que l'avenir, jamais le présent qui s'enfonce dans le passé. L'homme prend conscience du temps quand il projette de convertir un état actuel moins satisfaisant en un état futur plus satisfaisant ${ }^{8}$. Le temps est rare pour l'homme, il doit l'économiser comme les autres ressources rares, mais pour Von Mises le temps présente la particularité d'être irréversible (ce qui est évident) et unique (ce qui l'est moins). Il existe pour chacun une préférence temporelle subjective. «La préférence de temps est une nécessité catégorielle de l'action humaine ${ }^{9}$. Von Mises fait ici explicitement référence à un taux d'impatience subjectif qui est son prix psychologique du temps. Celui-ci ne doit pas être confondu avec le taux d'intérêt sur le marché, qui est le prix social du temps appelé " taux d'intérêt originaire » par Von Mises. C'est ce taux d'intérêt qui sert à arbitrer entre placement-investissement, d'une part, et consommation, d'autre part. Cette "économisation du temps est indépendante de l'économisation des services et des biens économiques. Même au pays de Cocagne,

\footnotetext{
${ }^{6}$ Ouvr. cit., chap 5, p.102-103.

${ }^{7}$ Ouvr. cit., chap. 5, p.103.

${ }^{8}$ Ouvr. cit., chap. 5 p. 100.

${ }^{9}$ Ouvr. cit. chap 18, p. 481.
} 
l'homme serait obligé d'économiser le temps, sauf s'il était immortel et doué de la jeunesse éternelle et d'une vigueur et d'une santé indestructibles ${ }^{10}$. L'analyse de Von Mises sur les facteurs individuels déterminant la préférence temporelle des individus est l'une des premières à faire référence à la durée de vie restante ou à la santé et à la jeunesse. La piste est ici ouverte, mais non exploitée, d'une évaluation du prix psychologique du temps variable dans le temps pour un même individu. C'est la première manifestation théorique de la possibilité d'une structure par terme des prix psychologique du temps dans la littérature.

\subsection{La théorie de l'utilité escomptée}

Samuelson [1937], à la suite des travaux de Ramsey [1928], développe une approche normative des choix intertemporels fondée sur un taux d'escompte constant. L'hypothèse centrale de la théorie de l'utilité escomptée est que l'agent économique rationnel maximise une séquence d'utilité intervenant à différentes périodes. L'axiomatique de Samuelson [1937] est fondée sur i) l'hypothèse d'intégration des utilités futures séquentielles aux décisions présentes - c'est-à-dire que l'individu décide globalement, ii) l'hypothèse d'indépendance de l'utilité à chaque instant - c'est-à-dire que la satisfaction de la consommation future ne dépend pas des choix antérieurs de consommation, et iii) l'hypothèse de stabilité de la fonction d'utilité instantanée, c'est-à-dire que les goûts de l'individu restent identiques ${ }^{11}$. Ces hypothèses imposent ainsi une cohérence intertemporelle des choix reposant sur la stabilité des préférences au cours du temps. En d'autres termes, les «mois » futurs respectent sans révision la réalisation des plans futurs initialement décidés par le «moi » présent. La rationalité économique de l'agent le conduit donc à optimiser son utilité totale sur son horizon décisionnel de vie.

$$
V_{t}=U\left\{C_{t}, C_{1} \ldots, C_{T} ; t, 1 \ldots T\right\}=\sum_{t=0}^{T} U\left(t ; C_{t}\right)=\sum_{t=0}^{T} d(t) . U\left(C_{t}\right)
$$

où $V_{t}$ définit l'utilité totale de l'horizon décisionnel de vie $T$ évaluée à la période courante $t$, et exprime le fait que le «moi $t$ prend les décisions. $C_{t}$ représente la variable de choix économique, traditionnellement la consommation globale en t. $d(t)$ représente le facteur d'actualisation temporel décroissant au cours du temps $\left(d^{\prime}(\mathrm{t})<0\right)$, car les conséquences futures envisagées sont sous-évaluées en raison d'une préférence pour le présent.

L'hypothèse d'une fonction d'utilité séparable temporellement («time separable») permet de faire ressortir la séquence des flux d'utilité instantanée $U$ intervenant à la période $t$. Cette hypothèse de séparabilité peut se concevoir sous une forme faible avec par exemple des fonctions d'utilité récursives. Une forme forte de séparation introduite par Samuelson conduit à une forme additive de la fonction d'utilité (cf. $2^{\text {ème }}$ terme de l'équation 1). En introduisant l'hypothèse de stationnarité, on obtient une séparation avec des fonctions d'utilité $U$ invariantes à chaque période et des facteurs d'actualisation associés. Les choix décisionnels de l'agent résultent alors de deux composantes distinctes relevant de l'utilité (notamment de l'aversion au risque) et de la dimension temporelle. Dans ce schéma, le facteur d'actualisation décroit à taux constant, c'est l'actualisation exponentielle:

\footnotetext{
${ }^{10}$ Ouvr. cit chap 5, p. 102.

${ }^{11}$ L'hypothèse de la stabilité de la fonction d'utilité instantanée (indépendante du temps) peut être élargie en considérant une fonction d'utilité qui évolue de manière aléatoire $U_{t+1}()=.U_{t}()+.\varepsilon_{t}$.
} 


$$
d(t)=\delta^{t} \text { avec } \delta \text { constant }<1
$$

Le taux de dépréciation du futur $d(t)$ se résume alors à un taux d'escompte subjectif constant, qui traduit tous les motifs individuels psychologiques conditionnant le caractère intertemporel des choix. La décomposition de l'utilité totale des plans de consommations futures (par une séquence d'utilités futures escomptées par un taux d'actualisation) permet ainsi de dissocier le taux de dépréciation du futur de la préférence temporelle. Samuelson [1937] ne considèrent pas la possibilité que le taux de dépréciation du futur puisse être variable au cours de l'horizon décisionnel de l'individu ${ }^{12}$. Un taux d'escompte non constant aurait ainsi permis d'identifier une fonction d'actualisation psychologique $\delta(t)$ propre aux préférences temporelles de l'agent.

Le modèle d'utilité escomptée de Samuelson respecte la condition de stationnarité les préférences intertemporelles sont les mêmes si l'instant auquel on se situe (ici $t=0$ ) change et si on se situe par exemple en $t+1$. On parle alors de rationalité intertemporelle stricte ou de choix dynamiquement cohérent. Koopmans [1960] discute l'axiomatique de l'utilité intertemporelle. Si on considère un horizon infini l'existence d'un taux d'impatience apparaît comme "une conséquence logique nécessaire des propriétés de la fonction d'utilité »(p. 306) », particulièrement celle del'hypothèse de structures de préférences stationnaires dans le temps et celle de capacité à agréger les utilités futures période par période.

\subsection{Le modèle standard d'évaluation financière: aversion au risque et substitution intertemporelle}

Le modèle micro-économique standard de l'investisseur-consommateur introduit l'incertitude et se situe dans un horizon temporel où le prix psychologique du temps est constant. L'agent économique raisonne en espérance d'utilités actuelles et futures. Les décisions sont prises sur la base d'une fonction d'actualisation personnelle qui est dès lors exponentielle. La fonction de consommation considère le plus souvent de la consommation agrégée d'un individu représentatif. Cette hypothèse supplémentaire rend les tests faciles car il est alors possible de prendre comme variable la consommation agrégée qui est une donnée mesurée.

Hansen et Singleton [1983] et Breeden [1986] ont formalisé l'équilibre consommation-investissement. Leur modèle d'évaluation considère la rentabilité espérée d'un actif risqué quelconque. L'agent représentatif est supposé présenter une fonction d'utilité puissance caractérisée par un coefficient $\alpha$ est le coefficient d'aversion relative au risque. L'hypothèse de distributions lognormales de la consommation et des rentabilités est aussi faite. En prenant en considération des horizons quelconque $t$, on introduit la gamme des taux d'intérêt purs $\left\{R_{f, t}\right\}$ en remarquant qu'il s'agit d'actifs sans risque. L'équivalent logarithmique de la structure par terme des taux d'intérêt est $\left\{r_{f, t}\right\}$, pour t variant de 1 à n s'exprime (les lettres en minuscule représentent les logarithmes et $\sigma$ la variance supposée non conditionnelles du processus de consommation) :

\footnotetext{
${ }^{12}$ Samuelson (1937) rejette le contenu normatif du modèle d'utilité escompté : « Toute connexion entre l'utilité telle que discutée ici et une analyse en terme de welfare est à nier (...) Il est encore complètement arbitraire de faire l'hypothèse que l'individu se comporte de telle sorte qu'il maximise l'intégrale de consommation de la forme envisagée (dans le modèle) », rapporté par Frederick et al. [2002].
} 


$$
r_{f, t}=-\log \delta+\frac{\alpha}{t} \cdot E_{0}\left(\Delta c_{t}\right)-\frac{1}{2 . t} \alpha^{2} \sigma_{c, t}^{2}
$$

Le taux d'intérêt sans risque est linéaire par rapport à la croissance de la consommation anticipée sur l'horizon, avec un coefficient de pente égal au coefficient d'aversion relative au risque $\alpha$. L'équation (3) peut être inversée pour exprimer la consommation espérée comme une fonction linéaire du taux sans risque avec une pente de $\psi=1 / \alpha$. Cette relation entre la croissance anticipée de la consommation et le taux d'intérêt définit aussi $\psi$ l'élasticité de substitution intertemporelle (EIS, «elasticity of intertemporal substitution »). Celle-ci mesure l'incidence d'une variation du taux d'intérêt sur la pente du chemin de consommation. La formulation de l'EIS « exponentiel» est celle qui découle d'un coefficient d'actualisation psychologique exponentiel fondé sur un taux de préférence constant au niveau de l'investisseur :

$$
E I S_{\exp }=\frac{\partial \log \left(C_{1} / C_{0}\right)}{\partial \log \left(R_{f, 1}\right)}=\frac{1}{\alpha}
$$

Dans le cadre d'une formalisation exponentielle standard de la fonction d'actualisation psychologique exponentielle (avec utilité additivement séparable et stationnaire), on met en évidence la relation directe (et inverse) entre EIS et $\alpha$, qui fait que les deux concepts se confondent. Le modèle micro-économique standard présente l'inconvénient majeur de confondre les concepts d'aversion au risque et d'élasticité de substitution intertemporelle puisque l'un est l'inverse de l'autre.

Les questions théoriques posées par le modèle de l'utilité actualisée standard sont nombreuses (voir Soman et al. [2005] ou Rohde [2010]). Au plan théorique, une première mise en cause du cadre d'analyse micro-économique standard a été faite par Epstein et Zin [1989]. Ceux-ci soulignent l'importance de la différence conceptuelle entre la notion d'aversion au risque et celle de substitution intertemporelle. Ils introduisent des systèmes de préférence récursifs et plus généraux que l'opérateur d'espérance d'utilité classiquement retenue dans la littérature : «Une caractéristique importante des préférences est que l'aversion au risque doit être distinguée du degré de substituabilité intertemporelle». La structure récursive des préférences permet de respecter la condition de cohérence temporelle. Epstein et Zin [1989] montrent en effet qu'il existe trois classes de fonctions satisfaisant cette condition dont la très classique espérance d'utilité additive. Cependant ils montrent aussi que dans la classe des fonctions d'utilité à la fois «time-separable » et additive, il n'est pas possible de dissocier les deux effets d'aversion au risque et de substitution intertemporelle. La fonction d'utilité récursive ainsi proposée par Epstein et Zin fait toujours référence à un facteur d'actualisation constant $\delta$. Epstein et Zin [1991], Weil [1989] conservent la propriété de «scale invariance » des fonctions d'utilité de forme puissance, mais leur modèle casse le lien entre l'aversion relative au risque et l'EIS. Les tests empiriques du modèle d'Epstein et Zin sont peu mitigés : ils tendent bien à valider l'idée d'un modèle avec des paramètres identifiant une substitution temporelle différents de ceux d'aversion au risque. Cependant des incohérences internes apparaissent avec les valeurs estimées des taux d'impatience psychologique négatifs (qui signifieraient des facteurs d'actualisation $\delta$ supérieurs à 1 ) et des valeurs estimées de l'aversion au risque parfois négatives ou parfois positives. Une autre voie d'évolution a consisté à modifier la représentation standard du modèle d'utilité actualisée en introduisant des habitudes de consommation. Le niveau de consommation actuel conditionne 
alors l'utilité marginale de la consommation future. Ces habitudes peuvent être internes ou externes. Les premières dépendent du comportement passé de l'individu. Les secondes dépendent d'un niveau global et exogène de consommation (Campbell et Cochrane, 1999).

\subsection{La piste d'une structure hyperbolique des prix psychologiques du temps et la contrainte de cohérence temporelle}

Une des premières voies de critique du modèle financier d'analyse des choix intertemporels a consisté à mettre en cause la fonction d'actualisation exponentielle, c'est à dire la constance du taux d'impatience. Il est proposé une structure des prix psychologiques du temps non constante et distincte du taux d'aversion au risque. Très vite l'idée d'une structure décroissante est apparue dans les évaluations empiriques. A ce titre, le test de Thaler [1981] constitue l'une des premières remises en cause empirique. Cette expérience consistait à demander à des individus "quel montant-dans respectivement 1 moi, 1 an et 10 ans-jugezvous équivalant à $15 \$$ ? ». La réponse médiane fut $20 \$$ à 1 mois, $50 \$$ à 1 an, $100 \$$ à 10 ans. Ce qui conduisit Thaler à établir une structure par terme décroissante des taux d'impatience dont les valeurs seraient de $345 \%$ sur 1 mois, $220 \%$ sur 1 an et $15 \%$ sur 10 ans.

L'interrogation directe sur la forme de la fonction d'actualisation psychologique de l'individu trouve encore son origine dans les expérimentations faites par des études de psychiatres ou de psychologues sur le comportement humain et animal. Chung et Herrnstein [1961] ont ainsi tiré de leurs expérimentations qu'une une fonction d'actualisation hyperbole stricte approxime la fonction de préférence temporelle d'animaux. Pour leurs parts, Ainslie [1992] et Loewenstein et Prelec [1992] proposent notamment un ajustement à l'aide de fonctions d'actualisation hyperboliques de forme générale $\delta(t)=\left(1+\alpha^{\prime} \cdot t\right)^{-\gamma / \alpha^{\prime}} .{ }^{13}$ Ces fonctions conduisent à des taux d'actualisation psychologique de la forme :

$$
-\frac{\frac{\partial}{\partial t}\left(\left(1+\alpha^{\prime} \cdot t\right)^{-\gamma / \alpha^{\prime}}\right)}{\left(1+\alpha^{\prime} \cdot t\right)^{-\gamma / \alpha^{\prime}}}=\frac{\gamma}{\left(1+\alpha^{\prime} \cdot t\right)}
$$

Ceux-ci sont décroissants dans le temps. Le taux d'intérêt psychologique à très court terme est égal au paramètre $\gamma$; à long terme, il converge vers zéro. Le lien entre la fonction d'actualisation et le taux d'escompte psychologique instantané est au point $t$ du futur donné par $-\delta^{\prime}(t) / \delta(t)$ où $\delta($.$) représente le coefficient d'actualisation psychologique. La fonction$ d'actualisation exponentielle traditionnellement utilisée en économie financière utilise un facteur $\delta(t)=\delta^{t}$, qui conduit à un taux d'actualisation psychologique annuel constant égal à $\ln (1 / \delta)$. La fonction d'actualisation hyperbolique conduit donc à un taux d'escompte psychologique décroissant avec l'horizon. Vu de la période 0 , le taux d'escompte psychologique entre $t$ et $t+1$ est un taux d'intérêt à long terme faible. Vu de la perspective de la période $t$, le taux d'actualisation entre $t$ et $t+l$ est au contraire un taux d'intérêt à court terme plus élevé que le précédent. Ces expérimentations suggèrent donc une actualisation hyperbolique, plus élevée pour le futur proche que pour le futur éloigné, en raison d'une surdépréciation du futur immédiat par rapport au futur éloigné. Ces représentations ont remettent en cause le modèle standard d'espérance d'utilité en raison d'un effet de différence

\footnotetext{
${ }^{13}$ Avec $\gamma$ et $\alpha^{\prime}$ comme paramètres. Une forme réduite a été proposée par Mazur [1987] qui pose $\gamma=\alpha^{\prime}$.
} 
commune, selon lequel les individus seraient plus sensibles à un délai temporel donné s’il advient plus tôt que plus tard.

L'enjeu théorique de telles fonctions est qu'elles conduisent à des séquences de choix économiques non consistants. Les choix optimaux effectués pour t à l'aide d'un facteur d'actualisation $\delta(\mathrm{t})$ ne sont pas les mêmes que ceux effectués une période plus tard actualisés à l'aide d'un facteur $\delta(\mathrm{t}-1)$. Ces conflits dans l'allocation dynamique des plans d'investissement-consommation ont été très tôt soulignés par Strotz [1956]. Celui-ci fut l'un des premiers à souligner que les agents sont plus impatients quand ils font des arbitrages économiques à court terme que lorsqu'ils font des choix économiques situés dans le long terme. En cas d'inconsistance dynamique des choix, l'allocation optimale s'analyse comme un conflit de jeux entre des agents différents : le «moi présent » décideur à l'instant $t$ entre en jeu stratégique avec le «moi futur » optimisateur en $t+1$. La perspective offerte par les fonctions hyperboliques consiste à dire que l'avenir à court terme compte plus dans la résolution des choix dynamiques que le long terme. A la limite, un individu complètement myope qui ne regarderait que l'instant présent et l'instant suivant n'a besoin que d'un taux d'actualisation entre $t$ et $t+1$, et donc n'est pas sensible au problème d'inconsistance dynamique de ses choix au-delà.

Strotz [1956] établit ainsi que la préférence pour le présent traduit une remise en cause de la cohérence temporelle des choix en raison d'une rationalité limitée de l'individu. D'autres analyses ont conduit au même constat: Akerlof [1991] considère un déficit d'imagination de l'individu dans la perception temporelle (effet de myopie), Laibson [1997] établit d'existence d'un déficit de volonté de l'individu dans la projection temporelle (effet d'impatience), O'Donoghue et Rabin [2001] mettent en évidence un déficit d'anticipation lié à la naïveté de l'individu. Ces études montrent ainsi l'existence d'une priorité du présent temporellement incohérente par le recours au «pré-engagement » ou la mise sous tutelle des «mois » futurs.

\subsection{Les propriétés de l'actualisation quasi-hyperbolique}

Il existe plusieurs modélisations du conflit entre les préférences du «moi » présent et celles du «moi » futur. Laibson [1996] a introduit une actualisation quasi-hyperbolique pour traduire l'existence d'un degré d'impatience plus fort à court terme respectant en même temps la contrainte de choix séquentiellement cohérents. La fonction d'actualisation quasihyperbolique de Laibson fait appel à un second paramètre $\beta$, en plus du coefficient d'actualisation constant standard $\delta$. Le coefficient d'actualisation chute au premier instant du futur par suite d'une multiplication $\operatorname{par} \beta<1$. A l'instant $t=1$, le coefficient d'actualisation est $\beta . \delta$. Les coefficients d'actualisation des périodes suivantes sont multipliés par le coefficient d'actualisation unitaire $\delta$; ce qui donne une fonction d'actualisation discrète $\left\{1, \beta . \delta, \beta \delta^{2}\right.$, $\left.\beta . \delta^{3} \ldots\right\}$. Laibson montre alors que dans une économie quasi-hyperbolique, l'EIS est plus faible que l'EIS « exponentiel»:

$$
E I S_{\text {quasi-hyperbolique }}=\frac{1}{\alpha}+\frac{1}{\alpha}\left[\frac{1}{\lambda *(\beta \delta-\delta)+\delta}\right] \cdot \frac{\partial \lambda *}{\partial \log (R)} \cdot(\beta \delta-\delta)
$$

où $\alpha$ est l'aversion au risque $(\alpha>0)$ et $\lambda^{*}$ une propension optimale à consommer. Avec un coefficient d'actualisation de la première période égal à $\beta . \delta$ et $\delta<1$, le second terme de l'équation (6) est de signe négatif (sachant que $\lambda^{*}<1$ et que $\beta<1$ ). L'EIS quasi-hyperbolique 
tend donc vers zéro pour des valeurs petites de $\beta^{14}$. Laibson [1996] utilise la fonction quasihyperbolique et montre, sous certaines conditions, qu'elle respecte la condition de consistance des choix dynamiques entre le «moi présent » en $t$ et le «moi futur» en $t+1$. Les choix faits par le même individu à deux instants différents du futur sont compatibles dans le cadre d'un comportement optimal pour chacun d'eux. La règle de comportement rationnel que ces deux «mois » doivent respecter est qu'à chaque instant, l'individu doit consommer une certaine proportion déterministe de sa richesse. La démonstration est possible car le taux de rentabilité $\mathrm{R}$ de l'épargne/investissement est supposé identique et constant quel que soit l'horizon. La mesure d'une EIS quasi-hyperbolique est ici problématique car les poids de la fonction d'actualisation sont discontinus ${ }^{15}$.

Un des apports de Laibson [1996] est aussi de souligner le risque d'erreur de spécification économétrique d'un modèle exponentiel faux alors que le vrai modèle de choix serait quasi-hyperbolique. Il montre, à partir de données macroéconomiques, que le facteur d'actualisation constant de 0,977 dans une monde exponentiel est, en terme d'estimation empirique, identique à une actualisation hyperbolique caractérisée par les valeurs $\beta=0,6$ et $\delta=0,99$. Un économètre peut ainsi à tort utiliser un taux d'impatience de l'ordre de $2,3 \%$ par an au lieu d'un taux correct de $1 \%$. L'erreur de choix sur le très long terme devient importante en raison de la fonction puissance. Toutes choses égales par ailleurs, le modèle exponentiel pénalise ainsi trop le long terme par rapport au moyen terme, alors que dans un monde exponentiel au-delà du court terme, les coûts d'attente marginaux sont plus ou moins stables entre le moyen et le long terme.

Laibson [1997] introduit, à partir d'une fonction d'actualisation quasi-hyperbolique, un modèle d'investissement consommation de l'individu sur des actifs illiquides, tel que le logement. Harris et Laibson [2001] proposent une démonstration théorique plus élargie des travaux de Laibson [1997], en reprenant une axiomatique mathématique plus générale qui montre la cohérence des choix dynamiques des consommateurs dits hyperboliques. Cependant le taux de rentabilité des actifs est toujours supposé constant et certain dans leur modèle. Dans un travail ultérieur, Harris et Laibson [2004] raisonnent en temps continu, ce qui conduit à faire disparaître les anomalies dans la forme de la fonction d'utilité de la consommation. Le raisonnement se situe à la limite en utilisant la notion de "gratification instantanée », c'est à dire que le taux d'impatience est très élevé à la limite de l'instant présent et de l'instant suivant. Il redevient constant pour l'ensemble du futur. La structure des prix psychologiques du temps présente donc un saut à l'instant 0 initial. Dans ce contexte, Harris et Laibson montrent l'existence d'un équilibre optimal unique pour les investisseurs-consommateurs.

Sorger [2003] développe un modèle en temps continu proche du précédent. Il distingue deux régimes d'épargne : à un moment donné l'investisseur bascule en s'engageant dans un processus d'épargne contraignant. Sorger introduit un temps d'arrêt qui est la période d'engagement des investisseurs-consommateurs. Plus cette variable est longue, plus la situation économique est améliorée sous forme d'un capital final supérieur par rapport à un consommateur-investisseur «éternellement jeune» («perpetual youth»). Il y a un gain d'optimalité à introduire la notion d'engagement d'épargne. Chez Sorger, les fonctions d'actualisation psychologiques des ménages ne sont pas forcément homogènes. A un moment donnée sur le marché, certains ont des taux d'actualisation élevé (c'est-à-dire qu'ils sont «éternellement jeunes » et se situent dans le cadre du futur proche) et d'autres sont dans la seconde période (ou futur lointain) de la courbe d'actualisation quasi-hyperbolique avec des

\footnotetext{
${ }^{14}$ Cf. Laibson, 1996, proposition 6.

${ }^{15}$ Nous remercions un rapporteur pour cette remarque.
} 
taux d'intérêt psychologiques bas. Le basculement entre les deux périodes est stochastique. Gollier et Zeckhausser [2005] ont repris l'idée de fonctions d'actualisation psychologique hétérogènes au niveau des ménages afin de déterminer la forme de la fonction d'actualisation psychologique collective agrégée permettant, par exemple, de prendre des décisions concernant des projets d'investissement en biens publics. Ils montrent que, sous certaines conditions, même si individuellement les individus ont des fonctions d'actualisation exponentielles avec pour chacune un prix du temps constant, la fonction d'actualisation d'un groupe d'agents hétérogènes n'est pas exponentielle. La fonction d'actualisation collective peut, sous certaines conditions, être de forme hyperbolique, c'est à dire qu'elle implique une structure des prix agrégés du temps décroissante. Ce résultat a aussi été mis en évidence par Lengwiller [2005] et par Nocetti, Jouini et Napp [2008] qui ont étendu ce résultat au cas où les taux d'actualisation individuels sont décroissants et stochastiques. Jouini et Napp [2010] établissent un lien avec la «cumulative prospect theory» de Tversky et Kahneman.

Une représentation au niveau individuel des choix intertemporels nécessite ainsi la prise en compte d'une structure temporelle des choix. Chaque individu agit sur tout l'horizon intertemporel de ses choix jusqu'à la disparition de son «moi économique ». Pourtant, le modèle standard considère un agent représentatif, qui contourne ce problème d'horizon temporel fini en raisonnant sur la durée infinie d'horizons cumulés. S'intéresser à la détermination des choix intertemporels et l'existence d'une structure individuelle des prix psychologique du temps pose le problème de l'horizon de vie de l'agent.

\section{Structure des prix psychologique du temps, rationalité et horizon de vie de l'agent}

La fonction de préférence temporelle signifie que l'individu pèse et compare l'utilité des instants futurs (au sens de la notion de la «pesée des plaisirs et des peines » de Bentham). Une première piste d'évolution est ouverte par les axiomes de la finance comportementale qui proposent une pesée différente des plaisirs et des peines. Cette idée consiste à donner une utilité non symétrique aux gains et aux pertes. D'après la théorie des perspectives («prospect theory ») de Kahneman et Tversky [1979], l'axiome d'ancrage détermine des fonctions d'utilité différentes de part et d'autre de l'utilité de référence. Une dissymétrie de la fonction d'actualisation est aussi évoquée par Thaler [1981], pour qui l'actualisation des gains et celle des pertes ne se ferait pas au même taux d'escompte psychologique. Les gains sont actualisés à un taux plus élevés que les pertes. Les individus semblent ainsi préférer des pertes immédiates plutôt que retardées. La dissymétrie de la fonction d'actualisation entraîne alors l'absence de symétrie de l'utilité des gains et des pertes futures.

Strotz [1956] identifie le conflit entre préférences actuelles et futures comme étant lié à des fonctions d'actualisation non exponentielles. D'autres sources d'incohérence peuvent découler de modifications de fonctions de préférence en général («changing tastes »). Il faut faire une distinction entre (a) la variation de l'utilité actuelle causée par un changement de l'utilité elle-même dans le temps et (b) la pure préférence pour le présent qui est le désir de ne pas retarder la consommation (Soman et al. [2005]). Harris and Laibson [2001b] ont montré que la valeur actuelle pour un consommateur dynamiquement incohérent avec des préférences hyperboliques peut être est la même que la valeur pour un autre consommateur dynamiquement rationnel avec des préférences exponentielles standards et une fonction d'utilité «state-dependant» du niveau de la richesse et non de la consommation. Prelec [2004] propose une mesure théorique de l'incohérence temporelle de l'agent («time 
inconsistency »), à partir du logarithme de sa fonction d'actualisation. Cette mesure construite dans la dimension de l'attitude face au temps établit un parallèle avec la mesure de l'aversion au risque de Pratt and Arrow, utilisée pour les choix dans la dimension du risque.

Les modèles à «mois » multiples sont une des voies pour contourner l'argument d'incohérence temporelle «time inconsistency » lié à des taux d'escompte psychologiques non constants. Ils sont évoqués à la fois par Thaler et Shrefrin [1981] ainsi que Laibson [1996]. Peu formalisés, ces modèles ne donnent pourtant pas lieu à des hypothèses testables en tant que telles. Thaler et Shrefrin [1981] analysent la compatibilité des choix temporels dans le cadre d'une théorie du pré-engagement «self-control». Ils identifient deux «mois » en conflit constitutifs de l'agent économique. L'individu à un moment donné du temps est supposé être à la fois un planificateur («planner») de ses choix d'investissementconsommation sur le long terme et un acteur agissant myope («doer»). L'acteur agissant optimise sur la seule prochaine période. Il existe alors un conflit entre ces deux préoccupations. Thaler et Shefrin [1981] proposent une analogie intéressante et explicite avec le conflit d'agence entre actionnaires et managers dans la firme. Le « self control » de l'agent est le moyen de réduire ce conflit entre le moi du planificateur et celui de l'acteur puisque l'individu possède deux ensembles de préférences en conflit à un moment donné du temps. Pour Thaler et Shefrin [1981], le seul moyen pour le planificateur d'agir sur l'acteur myope est de le contraindre soit en modifiant ses préférences, soit en lui imposant des règles de comportement et de choix. Les outils pour modifier les comportements prennent la forme d'incitations et de contraintes. Par exemple, concernant l'arbitrage consommation-épargne, les incitations pèsent sur l'agent myope en associant une valeur (morale) positive à l'épargne. Des règles d'auto-limitation ou d'auto-discipline sont aussi imaginables pour ne pas consommer (équivalentes à consommer des coupe-faims pour des individus qui s'astreignent à un régime alimentaire). D'autres méthodes considérées sont les règles obligatoires d'épargne sous forme de pourcentage planché du revenu, d'interdiction d'emprunter ou de plans de retraite obligatoires.

\subsection{Utilité décisionnelle et utilité indirecte}

Le modèle néo-classique de l'utilité subjective pose l'existence d'une structure de rationalité dans les choix de l'agent économique. Ce modèle de base trouve son origine historique chez Bentham. La psychologie de l'individu économique apparaît extrêmement frustre car elle est guidée par la recherche mécanique de satisfactions hédoniques. Cependant derrière la notion de plaisirs résultants, l'accent est mis sur le calcul entre les désutilités d'un choix alternatif qui implique des coûts ou des efforts immédiats pour des satisfactions immédiates ou futures. La logique est totalement rationnelle. Cependant, déjà chez Bentham, mais de manière plus nette chez Hayek ou Buchanan, la notion d'utilité rationnelle apparaît plus riche et moins mécaniste qu'un simple calcul «utilitariste » de stimulation. L'analyse néo-classique met en avant deux types d'utilité qui se conjuguent dans la fonction de satisfaction de l'individu. Kahneman reprendra cette distinction sur la base d'une approche psychologique dans la théorie des perspectives.

Le calcul des plaisirs et des peines conduit à l'édification d'une utilité cardinale qui est l'élément de base de l'action humaine. C'est «le souverain maître (qui) désigne ce que nous devrions faire et qui détermine ce que nous devons faire ${ }^{16}$. Cette approche lie l'utilité à la

\footnotetext{
${ }^{16}$ Bentham [1789], repris p.375 par Kahneman et al. [1997].
} 
décision. Les plaisirs et les peines encourues sont les conséquences, les résultats («outcomes ») de l'action de l'individu. Selon Bentham, «Un homme (...) regarde au-delà de l'événement qui est appelé son action, Il doit prendre en considération les conséquences de celle-ci; (...) et c'est uniquement de cette manière que l'idée de plaisirs et de peines donne naissance à la première ${ }^{17}$. Cette approche place donc la référence hédonique au cœur de l'action économique et au cœur de l'action humaine (Leloup, 2002). Cette logique de décision et de gestion est celle associée à l'utilité de décision («decision utility ») ou encore utilité directe au sens où un lien avec un choix économique coûteux est à la base de l'apparition certaine, s'il s'agit d'un bien immédiat acheté, ou incertaine s'il s'agit d'un bien futur. Pour Bentham, "Plaisirs et peines (sont) à la base de la naissance de toute action. Des plaisirs et des peines existent sans naissance d'une action, mais pas l'inverse ${ }^{18}$. Il existe donc une utilité source de plaisirs et peines qui ne trouve pas sa source directe dans des choix économiques, ni dans l'action de l'individu. Cette ouverture vers une « autre » source d'utilité ne conduit pas à un reniement de la théorie de la décision fondée sur la maximisation d'utilité. $\mathrm{Au}$ sens de Bentham, toute action économique couteuse relève bien de cette théorie qui a été opérationnalisée en univers incertain par Von Neuman et Morgenstern avec le critère de l'espérance d'utilité. L'introduction d'un espace entre l'utilité reçue et l'utilité provoquée n'est pas gênante pour la théorie de la décision économique. En revanche celle-ci devient incomplète pour comprendre les comportements et la rationalité de l'agent dès lors qu'une autre source d'utilité se manifeste. Schmidt [2006] insiste sur les origines de ce concept déjà présent chez les utilitaristes benthamiens, tels que Jevons et Edgeworth. Ces derniers se posent en effet la question «en amont» de l'utilité expérimentée ou ressentie. Kahneman, Wakker et Sarin [1997] rappellent ce que la "prospect theory » doit à cette intuition de l'utilité ressentie signalée par Bentham. Kahneman et Tversky ne se fondent pas sur les préférences des agents considérées comme données et définies sur l'ensemble des conséquences possibles de leurs décisions. (Schmidt, 2006, p. 6). Ils introduisent au contraire un espace entre les actions et l'utilité ressentie par les agents. Cet espace est celui d'une utilité «indirecte », qui est néanmoins perçue et reçue. Elle se combien à la précédente pour alimenter l'action et les comportements humains. On reviendra sur l'apport des psychologues cogniticiens modernes par la suite.

Hayek [1973,1979] critique d'emblée la vision strictement rationnelle de l'utilitarisme qui relie étroitement la satisfaction et les choix économique ${ }^{19}$. L'utilité pour Hayek est un attribut qui s'attache à des moyens économiques susceptibles d'usages efficaces («usefullness ») éventuels. Une évolution qu'il qualifie de désir rationaliste ou d'erreur constructiviste a fait selon lui changer le sens de l'utilité qui est devenue un attribut des fins visées (c'est-à-dire les plaisirs ou satisfaction engendrées). Sur ce point, il s'opposera notamment à Von Mises. Hayek critique l'utilitarisme limité à un calcul strict du bilan mécanique des plaisirs et des peines. Il fait remarquer que ce calcul «implique comme hypothèse que tous les effets particuliers sur les tiers d'un acte quelconque peuvent être connue de celui qui agit ${ }^{20}$. Ce n'est pas tant l'hypothèse de rationalité et d'information parfaite qui est ici critiquée que le fait que l'utilitarisme soit totalement centré sur l'acte et juge chaque action individuelle sur l'utilité de ses effets connus. Hayek oppose l'utilitarisme

\footnotetext{
${ }^{17}$ Bentham [1789, p. 99].

${ }^{18}$ Bentham [1813-1815, p. 11], rapporté par Leloup [2002, p. 417] : «Pleasure and pains, the basis of all the springs of action. Pleasures and pains exist without the springs; no vice versa »

${ }^{19}$ Hayek cite une phrase attribuée à Hume pour qui l'utilité est une «idée vague qui exprime l'aptitude à servir une intention, sans suggérer de relation stricte entre utilité et bonheur» (p. 351). L'intuition est conforme à celle de Bentham : le bonheur assimilable à l'utilité ressentie ne dépend pas directement d'une intention liée à une action éventuelle.

${ }^{20}$ Ouv. cit. p. 355.
} 
« générique » à l'utilitarisme direct lié à une décision particulière. L'utilitarisme générique est centré sur les « règles de conduite » qui intègrent dans l'analyse les effets collatéraux sur des tiers et les effets du long terme, c'est-à-dire du temps. Ce corps de règle, qui relève de la notion d' «ordre spontané » renvoi chez Hayek à la morale et au droit. Or, l'analyse utilitariste directe «élimine complètement l'ignorance dans la démarche rationnelle, c'est grave car c'est justement l'ignorance qui rend les règles de conduite nécessaires ${ }^{21}$. Les règles de conduite sont des guides de décision dans l'incertain. Elles complètent la rationalité limitée de l'acteur par des routines, des garde-fous ou des effets d'apprentissage qui sont efficaces socialement. "Comme la plupart des outils, les règles ne sont pas un élément d'un plan d'action, mais plutôt un équipement pour certains hasards inconnus $»^{22}$. Pour Hayek, il existe donc une utilité indirecte «sociale» liée à l'application de règles dans les choix économiques qui sont sources de bien-être social. Le modèle de rationalité utilitariste directe est incomplet notamment pour les effets à long terme.

L'analyse de Hayek reste cependant très générale et macroéconomique. Buchanan [1969] analyse les coûts dans la théorie des choix économiques. Fidèle à la tradition néoclassique, il rappelle que les coûts ont d'abord une dimension en terme d'utilité et non pas une dimension objective : "Dans une théorie des choix, les coûts représentent la perte d'utilité anticipée par rapport au sacrifice d'une alternative rejetée $»^{23}$. Le concept de coût permet de distinguer des «choice-influenced costs » et des «choice influencing costs ». La décision implique ces deux types de coûts. Les pertes d'utilité sont toujours des résultats de décision et jamais leur cause. Les coûts d'opportunités sont des sacrifices ex ante. Ce sont des obstacles aux choix. En tant que tel les coûts inhibent les choix économiques. Ces «choice influencing costs » sont des opportunités sacrifiées qui n'apparaissent pas dans la fonction d'utilité de l'agent économique. Le décideur supporte ex ante les «choice influencing costs », c'est à dire les profits ou coûts des alternatives délaissées. Ceux-ci disparaissent dès que le choix a été fait. Pour les coûts influencés par les choix, «le coût est relié au choix ex post, mais n'est pas personnellement lié à celui qui choisit ou au décideur $»^{24}$. Les résultats ne sont pas forcément supportés par ceux-ci. «Ceux qui portent le fardeau (...) n’ont pas nécessairement besoin d'être ceux qui supportent l'agonie du choix ${ }^{25}$. Dans ces conditions, il existe un écart systématique possible dans les coûts subjectifs intégrés dans le programme d'optimisation du décideur. La logique d'optimisation porte sur les coûts ex ante liés à la décision. Les coûts supportés sont autres. Buchanan ne pousse pas le raisonnement à ce niveau car cela conduirait à questionner la rationalité ex ante de la décision, mais l'inquiétant constat est que la fonction d'utilité rationnelle à respecter est celle des coûts finaux alors que la décision est basée sur des coûts alternatifs ex ante.

Kahneman [1994] introduit une distinction entre l'utilité de décision qui découle des choix, plus précisément de leurs conséquences et l'utilité ressentie («experienced utility »). La première est une représentation des préférences nécessaire pour calculer les décisions économiques individuelles. La seconde se situe au niveau du bien-être global de l'individu. La différence entre les deux formes d'utilité pose le problème de l'hypothèse d'intégration des décisions prises à une situation de bien-être visé préexistante. L'écart entre les deux formes d'utilité fait référence aux besoins et aux préoccupations psychologiques viscérales de l'individu (Frederick et al., 2002). Pour Kahneman et Tversky, il existe un filtre de

\footnotetext{
${ }^{21}$ Ouv. cit. p. 357.

${ }^{22}$ Ouv. cit. p. 362.

${ }^{23}$ Ouv. cit. p. 43.

${ }^{24}$ Ouvr. cit. p.48.

${ }^{25}$ Ouvr. cit. p.48.
} 
raisonnement que sont les expériences passées. Ce filtre induit un mécanisme anticipatif qui module les plaisirs et les désagréments futurs prenant en compte le temps et son effet sur les préférences des agents (Schmidt, 2006). Il existe des éléments d'utilité indirecte qui sont reçus ou dérivés de choix. Ils dépendent d'éléments de contexte, de la structure des choix antérieurs, d'aléas purs et des tensions entre les «mois» successifs qui forment une personnalité. Sur le plan théorique, la différence entre l'utilité de décision et l'utilité ressentie signifie que la fonction d'utilité instantanée peut ne pas être stable. Elle est modulée par le filtre des facteurs viscéraux de la psychologie humaine : la peur, la colère. L'objet de cet article est ainsi de monter qu'un des éléments de cette déformation pourrait être l'attitude face au temps. On retrouve ici des déterminants psychologiques complexes ou des facteurs sociologiques. Déjà, Fisher [1930] soulignait le rôle de la mode en conséquence de laquelle l'utilité d'un bien peut changer en tant que telle au cours du temps. Cette distinction entre l'utilité de décision et l'utilité ressentie est la même que celle existant entre ce que les individus font (rationnellement) et ce qui les rend heureux. C'est néanmoins dans le cadre limité de l'utilité de décision que s'est développé historiquement l'axiomatique de la rationalité des choix à la suite notamment des travaux de Von Neumann et Morgenstern. Ainsi que le fait remarquer Schmidt [2006], «la maximisation de l'utilité expérimentée ne coüncide pas nécessairement avec la maximisation des préférences sans pour autant remettre en cause l'idée d'une rationalité. Mais cette référence commune à la rationalité n'exclut pas des différences quant à sa conception » (p. 7). La principale conséquence de ce schéma est une définition moins exigeante de la rationalité et l'intégration notamment dans le temps de préférences qui ne sont pas forcément constantes.

\subsection{L'agrégation temporelle des préférences}

Dans l'approche comportementale, la séparabilité temporelle signifie que l'individu est capable d'identifier les utilités instantanées, à partir desquels il construit une utilité totale identifiée par «l'intégrale temporelle d'une sorte de transformation de l'utilité instantanée $»^{26}$. Le phénomène d'agrégation temporelle fait référence à une comparaison possible de deux utilités instantanées à deux moments différents de manière à identifier laquelle possède la plus grande valeur de plaisir hédonique. Kahneman et al. [1997] introduisent le terme de valeur, sans utiliser précisément le concept de coefficient d'actualisation ou celui de préférences temporelles subjectives. Cette «transformation » de l'utilité instantanée s'apparente ainsi à une notion de valeur actuelle (art. cit., p. 391) ; mais Kahneman et al. ne se posent pas la question de la fonction d'agrégation temporelle qui permet d'aboutir à une utilité totale cardinale.

L'axiome 3 de Kahneman et al. [1997] implique la séparabilité des utilités instantanées. Il est fondamental de cette manière que l'utilité instantanée devienne une «statistique suffisante» "en ce sens que toute l'information dont on a besoin pour évaluer les bienfaits d'un épisode peuvent être incorporés dans son profit d'utilité ${ }^{27}$. Par ailleurs, Kahnenam et Riis [2005, p. 8] reconnaissent que «le temps est la dernière ressource finie de la vie et que trouver le moyen de l'utiliser au mieux est un objectif d'importance à la fois pour l'individu concerné par son bien être qu'au niveau de choix sociaux concernés par le bien être humain ». Cela revient désormais à reconnaître le principe d'une valeur psychologique positive au temps, ce qui conduit aussi à se poser la question de la borne supérieure

\footnotetext{
${ }^{26}$ Cf. Kahneman et al. [1997, p. 388].

${ }^{27}$ Cf. Kahneman et al. [1997, p. 390].
} 
d'agrégation des utilités futures. La valeur psychologique de temps s'apparente ainsi à l'évaluation subjective du temps en fonction de la durée de vie ; elle est plus large que le strict taux d'escompte psychologique qui porte seulement sur des arbitrages de choix économiques. La finitude de la vie pose donc le problème de l'identité de l'individu distincte de celle de ses successeurs. Cette identité économique est d'abord la conscience de la stabilité des goûts de l'agent : suis-je le même qu'hier ?, serai-je demain le même qu'aujourd'hui ? La question de la borne supérieure d'évaluation de l'utilité et du bien-être pose le problème de l'interruption des «mois » successifs inscrits dans une logique d'utilité de décision par la mort de l'agent. Les successeurs de l'agent (ses héritiers, la société au sens large...) ne sont plus des «mois » économiques. L'identité de l'acteur s'arrête alors pour laisser place à l'identité d'autres acteurs et à leurs choix. Cela ne signifie pas pour autant que l'horizon d'évaluation soit borné par l'horizon de vie.

En ignorant la dissymétrie des plaisirs et des peines, le programme de l'acteur économique à partir de la spécification d'une fonction d'utilité variable dans le temps s'écrit ${ }^{28}$ :

$$
\Omega=E_{0}\left[\sum_{t=0}^{\infty} \delta(t)^{t} U\left(t, C_{t}\right)\right]
$$

avec $\delta(t)$ : structure par terme de préférence temporelle

Les agents économiques sont tenus d'être cohérents dans leurs choix. Cependant on privilégie ici le principe d'une rationalité limitée, qui reconnait des conflits possibles entre les mois successifs de l'individu dans le temps. Ces choix s'entendent en termes global d'utilité ressentie. La notion de cohérence n'est pas celle de rationalité infinie de l'utilité décisionnelle conduisant à des choix stationnaires et dynamiquement cohérents. La cohérence signifie l'existence d'une logique de réduction des conflits dans le respect de certains principes. Un de ceux-là est celui de la préférence temporelle pour le présent.

La notion de fonction de préférence temporelle signifie ici qu'il existe un arbitrage psychologique possible entre un plaisir (ou une peine) immédiat et un plaisir (ou une peine) future. L'individu rationnel considère l'espérance des utilités actualisées $\Omega$. Cette définition d'un individu rationnel calculateur dont la fonction d'utilité est temporellement séparable « time separable » est soutenue par Becker et Murphy [1988], pour qui un individu qui ignore les conséquences futures à très long terme de ses actes, n'est pas rationnel : "Bien qu'un comportement totalement myope soit formellement cohérent avec notre définition du comportement rationnel, une personne qui négligerait entièrement ou principalement les conséquences futures de ses actes peut-elle être appelée rationnelle? ${ }^{29}$. Une réponse peut être trouvée en mettant en cause le schéma de l'agent représentatif ayant une durée de vie infinie.

Une autre critique de l'horizon temporel infini de l'action humaine provient d'auteurs sociologues ou psychologues qui considèrent que la vie économique s'inscrit dans un tableau temporel unifié qu'il faut considérer comme un tout. On ne peut dès lors distinguer un élément de ce tableau, par exemple l'espérance d'utilité perçue en $t$, du reste. Même si une illusion cognitive fait que tel ou tel élément du tableau temporel est plus ou moins perçu

\footnotetext{
${ }^{28}$ Le programme $\Omega$ est simplifié si est faite l'hypothèse d'une stabilité de la fonction d'utilité.

${ }^{29}$ Cf. art. cit. p.684.
} 
comme important et/ou que le long terme est par exemple moins valorisé, tous les éléments comptent. Il n'y a pas d'arbitrage entre plaisir (ou peine) dans le temps. Le recours au concept de fonction d'actualisation psychologique est donc rejeté, en revanche les utilités futures restent anticipées.

$$
\Omega=\left\{C_{0} ; E_{0}\left[U\left(C_{1}\right)\right] ; E_{0}\left[U\left(C_{2}\right)\right] ; \ldots E_{0}\left[U\left(C_{t}\right)\right] ; \ldots\right\}
$$

Dans le programme (8), il est irrationnel de préférer un plus petit plaisir immédiat par rapport à un plus grand plaisir futur car maintenant et plus tard font également et indépendamment partie de la vie. L'agent optimise une séquence initiale de choix juxtaposés. Pour Rawls [1971], "la rationalité exige une prise en considération impartiale de toutes les composantes de la vie. La simple différence de positionnement dans le temps de quelque chose qui est plus tôt ou plus tard, n'est pas une raison suffisante pour avoir plus ou moins de considération pour elle ${ }^{30}$.

L'apport de la théorie économique standard est ici de montrer que la conception holiste d'un individu optimisant globalement son tableau temporel négligerait la «time separability » de ses choix, c'est à dire le séquencement de décisions plus ou moins liés. La profondeur temporelle de l'action économique est fondée sur l'articulation d'une séquence de «mois » agissants. Un agent économique est une succession de «mois » qui se chevauchent dans l'action. Le point de passage d'un «moi » en $t$ à un «moi » en $t+l$ est une série de choix (ou de non choix) qui lui donnent accès à une série d'utilités futures, fruits de ses décisions («decision utility»). Ces «mois» sont liés entre eux par des phénomènes de mémoire, une continuité physique, des similarités de caractère et d'intérêts. Ils sont néanmoins différents. C'est dans ce contexte, que s'appréhende la déformation des fonctions d'utilité qui évoluent dans le temps et se modifient tout au long de la vie. Le moteur le plus traditionnel de cette déformation est l'évolution de la richesse. Toutefois, il faut aussi intégrer les éléments de continuité et de différenciation des «mois » successifs. Par exemple, l'utilité de manger un $2^{\text {ème }}$ beefsteak est grande quand on est jeune et doté d'un solide appétit, l'utilité d'un $2^{\text {ème }}$ beefsteak quand, avec l'âge, on a moins d'appétit et plus aucune dent, est nettement plus faible.

Il y a autant d'éléments de différenciation et de continuité entre les «mois » du même agent dans le temps qu'entre des agents différents, mais contigus vivants au sein d'une même société. Dans l'analyse des comportements économiques, l'actualisation des préférences temporelles est donc aussi rationnelle que l'agrégation des agents. Pour Frederick [2006] : «Il peut être tout autant rationnel d'actualiser l'utilité future d'un moi agissant de quelqu'un que d'actualiser l'utilité d'un autre individu car la différence entre les étapes d'une vie de quelqu'un peut être aussi grande que la différence entre (les actions) comparée entre individus différents ${ }^{31}$.

A cette étape, la question induite est celle de la cohérence des choix séquentiels entre les différents «mois ». La condition de cohérence temporelle «time consistency» se pose donc lorsqu'un prix psychologique non constant est retenu. L'avantage technique d'un facteur d'actualisation $\delta$ constant est surtout d'aboutir à une considérable simplification du problème d'investissement-consommation de l'agent économique standard. Cette hypothèse permet de plus d'éviter toute incohérence dans la séquence de choix temporels ente les différents

\footnotetext{
${ }^{30}$ Cf. art. cit. p.293.

${ }^{31}$ Cf. art cit. p.674.
} 
«mois ». Un des premiers enjeux théoriques de l'existence d'une structure par terme différenciée du prix psychologique du temps est donc le rejet de l'hypothèse de cohérence temporelle des choix [Strotz, 1956]. Ce point conduit à réfléchir sur la notion de rationalité des choix temporels. La préférence pour le présent se voit accorder un rôle crucial dans les choix intertemporels, mais elle traduit cette fois une rationalité limitée par un déficit d'imagination - « myopie »- ou de volonté - «impatience ».Ce déficit conduit le plus souvent à un problème d'incohérence temporelle des choix, c'est-à-dire de conflit entre les désirs du moi présent et du moi futur, entre les préférences d'aujourd'hui et de demain. Akerlof [1991] attribue l'incohérence temporelle plutôt à un déficit d'imagination et Laibson [1996, 1997] plutôt à un déficit de la volonté (Arrondel et al., 2004)

\subsection{L'horizon de vie et la préférence intergénérationnelle}

Le problème de la cohérence temporelle n'est qu'un des aspects de la rationalité de l'agent. Il se manifeste quand on raisonne sur un agent représentatif, notamment, à durée de vie infinie. C'est bien là une des limites de l'analyse théorique par rapport à une analyse individuelle des choix d'un individu qui ne peut exprimer une perception de son utilité dans le temps que sur sa durée de vie. La fonction d'actualisation individuelle est une donnée psychologique personnelle, qui bute à long terme sur la question de la désirabilité du transfert intergénérationnel. Un agent représentatif a durée de vie infinie ignore ce problème car chaque élément de l'utilité de consommation ou de richesse futur est pondéré par la probabilité subjective que l'agent soit en vie pour en profiter et l'intégrer dans son calcul. L'horizon est celui de la vie probable.

Il faut ici réintroduire la notion d'utilité vécue et non plus produite (« experienced utility» vs «decision utility») qui fait référence aux qualités hédoniques issues de l'expérience. Les plaisirs et les peines peuvent découler de décisions, ils peuvent aussi procéder de situations indépendantes et ne pas être liées à l' «impetus » économique de consommation ou de richesse produites par des décisions (Frederick, 2006). La rationalité de l'action n'est pas le seul pourvoyeur d'utilité, l'utilité perçue est plus large comme l'a initialement signalé Bentham car elle intègre la dimension hédonique de l'expérience. La pesée des plaisirs et des peines prend alors un sens global au-delà des actions humaines soumises à une rationalité de choix. Dans ces conditions, Kahneman et al. [1997] proposent une axiomatique des comportements individuels en remontant à la notion d'utilité vécue («experienced utility »), qui dépasse la logique simple de l'utilité calculée. Cette utilité vécue se situe dans un cadre de référence qui fait appel à l'introspection et à l'appréhension rétrospective des choix, des plaisirs et des peines passées (Kahneman et al., 1997 ; Kahneman et Riis, 2005). L'utilité vécue est par conséquent de nature différente de l'utilité calculée. Elle n'est pas liée à un cadre temporel. Elle n'est pas uniquement endogène à un cadre décisionnel. Elle «tombe» de manière en partie exogène sur l'individu. Elle surgit d'un processus introspectif et rétrospectif complexe. Pour Kahneman et al. [1997], «à la différence (de l'utilité de décision), l'utilité vécue totale d'un résultat n'est pas évaluée à partir d'un point particulier $d u$ temps ». Cette analyse ne remet toutefois pas en cause la nécessité rationnelle d'une actualisation de l'utilité dans les choix économiques.

Le cas le plus général serait celui d'un agent actualisant l'utilité future de ses décisions (qui lui confèrent une consommation décalée) et l'utilité ressentie (qui « tombe » sur l'agent comme largement issue d'évènements extérieurs). A ce titre, le premier exemple d'évènement ressenti et non maîtrisé est la mort. A chaque instant, une probabilité de mort vient transmettre 
le capital accumulé par l'agent à la génération suivante. Ce transfert procure une utilité indirecte vécue $U_{i, G}$ de nature différente de celle de l'utilité de décision. Le poids donnée à cette utilité future n'a donc a priori aucune raison d'être le même que celui donnée à l'utilité future décidée, $\delta(t)$.

$$
\Omega_{i}=E_{0}\left[\sum_{t=0}^{T} \pi_{i}(0, t) \cdot \delta_{i}(t)^{t} U_{i}\left(C_{i, t}\right)+\sum_{t=1}^{T}(1-\pi(0, t)) \eta_{i}(t+1)^{t+1} \cdot U_{i, G}\left(W_{i, t+1}\right)\right]
$$

avec $T$ : horizon de vie de l'agent et $\pi_{i}(0, t)$ : probabilité psychologique de survie de l'agent $i$ jusqu'à $t$.

Le concept de prix psychologique du temps ne concerne que le seul bien être de l'agent. L'agent peut-il actualiser le temps des autres qui lui succèderont, par exemple les héritiers. A notre avis, la réponse est négative. Il peut se sentir concerné par la richesse transmise aux autres, mais il ne peut projeter sa place et actualiser l'utilité future des autres. C'est pourquoi il est nécessaire d'introduire la notion de valeur psychologique du temps, qui porte sur l'utilité vécue «experienced utility » alors que le prix psychologique du temps sert pour l'évaluation de l'utilité calculée «decision utility» résultant des choix intertemporels.

Sur ce point l'analyse de Frederick [2006] fait une confusion entre l'actualisation de l'utilité future pour autrui et l'utilité personnelle de la transmission de sa richesse future au profit d'autrui. La richesse générée par la suite après sa mort ne concerne plus l'individu et ne peut donc être actualisée comme de la consommation ou de la richesse intra-générationnelle. Certains individus sont très sensibles, en ce qui les concerne, de savoir si l'utilité future qui leur profitera est proche ou éloignée dans le temps. Ils ont donc une préférence temporelle très marquée alors qu'en même temps ils n'ont aucune implication sur l'utilité future de ceux qui suivront après leur mort, ou inversement. Tous les cas de figure sont ainsi possibles. Le programme (9) pose d'abord une question empirique relative à la sensibilité des agents à l'utilité des générations futures. C'est aussi une question éthique pour laquelle les individus doivent-ils être sensibles dans leurs choix personnels à l'utilité future des nouvelles générations. Ces questions portent sur la fonction $U_{i, G}$ représentant l'utilité de transmission de richesses intergénérationnelles (Arrondel et al., 2004). Cette fonction d'utilité n'a rien à voir avec $U_{j}$, l'utilité future de la consommation des générations suivantes. Cette utilité bien particulière $U_{i, G}$ dépend du temps de mort anticipé. Elle n'est donc pas actualisée de la même manière que les utilités futures de l'individu car le sens de la préférence temporelle n'est pas le même. Il y a impatience à consommer pendant sa vie, mais pas à mourir.

Selon Frederick [2006], il faut à minima faire intervenir un taux d'actualisation psychologique différent $\eta($.$) assimilable à une valeur psychologique du temps. Pour$ Kahneman et al. [1997], il n'y a pas lieu d'appliquer une valorisation temporelle à cette utilité car «la neutralité temporelle apparaît la plus appropriée pour évaluer l'utilité vécue (experienced utility) » ${ }^{32}$. On a alors $\eta()=$.1 ; il n'y a pas d'impatience à mourir. L'heure de la mort est prise comme une fatalité que l'individu ne juge pas. On peut imaginer des cas de figure complémentaires si l'individu est suicidaire et souhaite hâter sa disparition; il aura

\footnotetext{
${ }^{32}$ Cf. art. cit. p. 393. La position de Kahneman et al. (1997) est cependant largement normative. Ils argumentent la neutralité au temps de l'utilité vécue comme permettant justement d'éviter l'actualisation qui introduit un biais systématique à l'encontre de conséquences à long terme défavorables par rapport à des bénéfices immédiats. «La time neutrality est judicieuse à la fois en tant que règle de prudence personnelle et en tant que principe dans les choix publics » (art. cit., p. 393).
} 
alors une fonction de préférence temporelle $\eta(\mathrm{t})$ positive $(\eta(\mathrm{t})<0)$. Symétriquement, on peut imaginer que des individus désireux de transmettre un capital aux générations suivantes souhaitent cependant rester en vie le plus longtemps possible. Ceci conduirait alors à considérer une fonction de préférence temporelle de la transmission intergénérationnelle négative, qui capitalise l'utilité future au lieu de l'actualiser $\eta(\mathrm{t})>1$. Il y a donc une série de trois prix du temps: les deux prix psychologiques des agents liés de manière intergénérationnelle $\delta_{i}$ et $\delta_{j}$ (qui actualisent les utilités de décisions) et un troisième prix $\eta_{i}($. (qui actualise un résidu de capital comme une utilité de type hédonique et ressentie). Ces sources d'utilité ne sont ni recherchées, ni décidées; elles ne font pas l'objet de choix économiques dans le temps, mais elles sont contingentes. Une attitude fataliste neutre conduit alors à poser $\eta_{i}()=$.1 . Le programme d'optimisation intertemporel de l'agent devient :

$$
\begin{aligned}
& \Omega_{i}=E_{0}\left[\sum_{t=0}^{T} \pi_{i}(0, t) \cdot \delta_{i}(t)^{t} U_{i}\left(C_{i, t}\right)+\sum_{t=1}^{T}(1-\pi(0, t)) U_{i, G}\left(W_{i, t+1}\right)\right] \\
& \Omega_{i}=E_{0}\left[\sum_{t=0}^{T} \pi_{i}(0, t) \cdot \delta_{i}(t)^{t} U_{i}\left(C_{i, t}\right)+\bar{U}_{G}(0, \pi(.))\right]
\end{aligned}
$$

Le facteur d'utilité intergénérationnelle cumulé $\bar{U}_{G}$ est une source d'incohérence temporelle car il valorise différemment dans le temps les entrées de consommation dans la fonction d'utilité $U(\mathrm{C})$ et les sorties liées à l'utilité de la richesse transmise en cas de mort.

Considérons la séquence d'utilités futures A $(0,8 ; 0,4 ; 0,65)$ et $\mathrm{B}(0,8 ; 0,45 ; 0,6)$ sur 3 périodes. La probabilité de survie est de $100 \%$ en début de période de choix, c'est-à-dire à la période 0 en se situant en $\mathrm{t}=0$. Elle est de $50 \%$ pour le début de période 1 et de $10 \%$ en début de période 2. Le facteur d'actualisation constant par période est 0,8 . L'utilité actualisée de la consommation future est clairement en faveur de $B$ en se situant en $t=0$. En se situant en $t=1$ avec une probabilité de survie qui est de $20 \%$ pour $\mathrm{t}=2$, l'utilité actualisée de la consommation est aussi en faveur du scénario $\mathrm{B}$ réduit à deux utilités. Il y a donc consistance temporelle des choix de consommation stricto sensu. La première utilité $U_{0}$ ne crée aucune distorsion, car elle est identique dans les deux scénarios $\mathrm{A}$ et $\mathrm{B}$. En se situant en $\mathrm{t}=0$, en anticipant et en actualisant le choix rationnel de $U_{C}$, on obtient bien la même valeur actuelle de l'utilité de décision de $\mathrm{B}$ qui est de 1,018. Si on intègre une utilité intergénérationnelle $U_{G}$ égale à la consommation reportée sur autrui en cas de mort, l'utilité de la richesse transmissible est, de plus, supposée égale à l'utilité de la richesse qui aurait été consommée en cas de vie. L'utilité $U_{G}$ est plus grande en se situant en $\mathrm{t}=0$ dans le cas du scénario A ; l'utilité totale $V$ de l'agent en intégrant l'utilité intergénérationnelle bascule en faveur du scénario A. La prise en compte d'une utilité élargie et ressentie conduit donc à des choix différents de ceux qui découleraient d'une stricte évaluation de l'utilité décisionnelle. Ce n'est pas en soi un résultat innovant.

En se situant en $\mathrm{t}=1$, l'intégration de l'utilité transgénérationnelle de la richesse transmise est plus faible en valeur absolue et est toujours en faveur du scénario A $(0,52$ contre $0,48)$. A cette date le scénario optimisant l'utilité ressentie totale est en faveur du scénario B (du moins en faveur des deux dernières utilités $U_{1}$ et $U_{2}$ de ce scénario). Il n'y a pas cohérence temporelle des choix par rapport à la séquence optimale des utilités définies en $\mathrm{t}=0$, qui privilégie le scénario A. Pour compléter la démonstration, il faut se situer en $\mathrm{t}=0$ et considérer des scénarios A ou B complets et alternatifs. En se situant en $\mathrm{t}=0$, il faut intégrer la première utilité, qui est identique dans les deux scénarios. L'utilité totale $V$ du scénario B intégrant la meilleure décision pour $\mathrm{t}=1$ est de 1,483 . Elle reste supérieure. Il n'y a alors pas cohérence temporelle des choix. Cependant, elle ne modifie pas le choix en faveur de B. Cet exemple 
montre qu'une inconsistance temporelle des choix peut trouver une cause supplémentaire en intégrant une utilité intergénérationnelle ${ }^{33}$.

Tableau 1 - Exemple de choix en cas de prise en compte de l'utilité intergénérationnelle (Choix séquentiels en situation d'utilité de décision $U_{C}$ et en présence d'utilité intergénérationnelle $\bar{U}_{G}\left(V=U_{C}+\bar{U}_{G}\right.$ : utilité totale ressentie; coefficient d'actualisation psychologique constant $\delta=0,8$; utilité intergénérationnelle non actualisée)

\begin{tabular}{|c|c|c|c|c|c|c|c|c|c|}
\hline & Utilités & $U_{0}$ & $U_{1}$ & $U_{2}$ & & & & & \\
\hline en $t=0$ & $\begin{array}{c}\text { Probabi } \\
\text { lité de } \\
\text { survie }\end{array}$ & $100 \%$ & $50 \%$ & $10 \%$ & $U_{C}$ & $\bar{U}_{G}$ & $V$ total & Choix $U_{C}$ & Choix V \\
\hline & A & 0,8 & 0,4 & 0,65 & 1,002 & 0,785 & 1,787 & $\mathrm{~B}$ & A \\
\hline & B & 0,8 & 0,45 & 0,6 & 1,018 & 0,765 & 1,783 & & \\
\hline en $t=1$ & $\begin{array}{c}\text { Probabi } \\
\text { lité de } \\
\text { survie }\end{array}$ & & & $20 \%$ & & & & & \\
\hline & A & & 0,4 & 0,65 & 0,504 & 0,520 & 1,024 & $\mathrm{~B}\left(\mathrm{U}_{1}, \mathrm{U}_{2}\right)$ & $\mathrm{B}\left(\mathrm{U}_{1}, \mathrm{U}_{2}\right)$ \\
\hline $\begin{array}{c}\text { en } \mathrm{t}=0 \\
\text { pour } \\
\mathrm{t}=1\end{array}$ & $\begin{array}{c}\text { Probabi } \\
\text { lité de } \\
\text { survie }\end{array}$ & & 0,45 & 0,6 & 0,546 & 0,480 & 1,026 & & \\
\hline & A & 0,8 & 0,504 & & 1,002 & 0,460 & 1,462 & $\mathrm{~B}$ & $\mathrm{~B}$ \\
\hline & B & 0,8 & 0,546 & & 1,018 & 0,465 & 1,483 & & \\
\hline
\end{tabular}

L'incohérence temporelle des choix ne signifie pas une irrationalité globale mais simplement une rationalité locale du moi limitée à l'instant $\mathrm{t}$. Il y a réconciliation entre une rationalité temporelle locale et rationalité globale au début de la période $T$ précédant la mort car le choix est simple et forcément cohérent entre une dernière consommation et la transmission de la richesse. Enfin, une dernière raison, autre que celle d'un prix psychologique du temps non constant, peut expliquer l'apparition d'une inconsistance temporelle des choix. Les fonctions d'utilité peuvent dans leur forme même dépendre du temps $U=U\left(t, C_{t}\right)$ en faisant l'hypothèse de changement des préférences «changing tastes ». C'est dire que la fonction d'utilité de l'individu évolue dans le temps en fonction de sa richesse, de son histoire, de ses satisfactions ou peines cumulées et/ou insatisfaites, de sa mémoire et de ses espoirs (Frederick et al., 2002).

Retenons donc que l'élément de perception et d'évaluation est l'individu. On se situe dans le cadre traditionnel de l'individualisme méthodologique. Une méthodologie privilégiant les données et mesures individuelles s'impose donc. L'individu est rationnel au sens d'une rationalité limitée et instantanée dans un contexte de choix qui appartient au couple (individu, temps). Cette rationalité locale ne correspond pas à l'exigence d'une rationalité absolue qui imposerait la condition extrêmement exigeante ex ante d'une consistance temporelle de la chaîne des choix actuels et futurs. Par ailleurs, l'individu peut présenter une fonction de

\footnotetext{
${ }^{33}$ Alors même que les choix fondés sur une utilité décisionnelle standard sont cohérents dans le temps. Cet exemple peut être généralisé en faisant intervenir explicitement des formes de fonction d'utilité $U($.$) et U_{G}($. différentes, ainsi qu'une fonction $\eta($.) différente de $\delta()$.1 .
} 
préférence temporelle complexe qu'il applique pour calculer l'équivalent subjectif actuel de l'utilité future. L'équivalent subjectif actuel fait référence à la probabilité que le «moi » futur n'existe plus, c'est à dire à sa probabilité subjective de survie/mort. La perception du futur est complexe au niveau individuel car elle est le mélange de deux variables $\pi_{i}(t, j)$ et $\delta_{i}(j)$. L'explicitation de la fonction de préférence temporelle pure impose donc de déflater l'expression des préférences de la probabilité subjective de survie qui est au cœur du programme d'un individu à durée de vie finie qui possède une utilité intergénérationnelle.

\section{Les évaluations empiriques}

Les tests empiriques de la valeur du prix psychologique du temps sont relativement nombreux ; ils ont été décrits dans l'article récapitulatif de Frederick et al. [2002].

\subsection{Les études sur données réelles}

Un première approche retenues dans les évaluations empiriques a consisté à partir de données réelles le plus souvent agrégées pour expliciter le facteur d'actualisation psychologique, en estimant les modèles d'équilibre financier en particulier le «Consumption Capital Asset Pricing Model »(CCAPM). Le test empirique du C-CAPM appliqué en considérant des données hors inflation a conduit au «puzzle du taux sans risque » identifié par Weil [1989]. Sur données américaines, le taux d'intérêt à court terme réel ressort en moyenne à $1,8 \%$. La croissance moyenne de la consommation réelle est estimée à $1,7 \%$ et l'écart type de la croissance de la consommation à 3,3\%, pour un coefficient d'aversion au risque de 19 mis en évidence par Merha et Prescott [1985]. Ces derniers chiffres conduisent à un facteur d'actualisation psychologique de 1,12 impliquant alors une préférence temporelle négative. Celle-ci est contraire au principe même de la préférence temporelle.

Hansen et Singleton [1982] utilisent la méthode des moments généralisés avec des variables instrumentales pour estimer l'aversion au risque et le taux d'escompte psychologique. Sur données américaines issues de marchés d'actions, ils aboutissent à des estimations de $\delta$ de l'ordre de 0,996 à 0,999 pour des fréquences mensuelles. Cela correspond à des taux d'escompte psychologique positifs de $1,2 \%$ à $4,8 \%$ pour un horizon de 1 an. Campbell et Cochrane [1999] ont calibré leur modèle de consommation sur des données historiques américaines. En supposant une aversion au risque de 2 et un taux sans risque constant, Campbell et Cochrane [1999] obtiennent un coefficient $\delta$ supposé constant de 0,89 (soit un taux d'intérêt psychologique de 12\%). Ces derniers auteurs montrent à partir de ces résultats que leur modèle permet de résoudre le puzzle du taux sans risque. Cocco et al. [2005] étudient aussi les choix de consommation et de structure de portefeuille sur le cycle de vie en présence de revenus de travail aléatoires. Ceux-ci sont non négociables ou assurables et expriment la rentabilité du capital humain. La prise en compte de cette variable influence la forme de la structure des prix psychologiques du temps.

Harris et Laibson [2001] ont calibré sur les données des ménages américains les deux structures de taux d'intérêt psychologiques constants (pour les «ménages exponentiels ») et décroissants (pour les «ménages hyperboliques»). Les «ménages hyperboliques» investissent une partie plus limitée de leur richesse dans des actifs liquides que les «ménages exponentiels ». Ce résultat apparaît en analysant aussi bien les actifs liquides en proportion des revenus du travail qu'en proportion de la richesse totale. En simulant les données, 40\% des «ménages hyperboliques» ont des actifs liquides supérieurs à 1 mois de revenu du 
travail ; ce pourcentage monte à $73 \%$ pour les «ménages exponentiels ». Les données réelles donnent une proportion de $42 \%$, qui est cohérente avec la moins grande appétence pour les actifs liquides (cf. tableau 2). Les «ménages hyperboliques» apparaissent aussi beaucoup plus emprunteurs sous forme de crédit pour la consommation personnelle (mesurés par l'encours de cartes de crédit). D'après les simulations, $51 \%$ y ont recours contre $19 \%$ pour les «consommateurs exponentiels ». Ce point est cohérent avec un taux d'impatience à court terme très élevé. Dans la mesure où les «ménages hyperboliques » ont un plus faible niveau d'actifs liquides et un plus fort niveau de dettes, ils sont moins capables que les autres de lisser leur dynamique de consommation en présence de chocs aléatoires liés à des revenus du travail incertains. La simulation des données calibrées entre les deux catégories de comportement des ménages montre aussi une plus forte corrélation entre la consommation totale et les revenus du travail prévisibles, correspondant bien à la corrélation empiriquement constatée. A l'inverse, la consommation calibrée dans le cas d'un «investisseur exponentiel» apparaît trop faible.

Tableau 2 - Données d'épargne américaines : Enquête «personal income » et encours d'emprunts en $\$ 1990$

\begin{tabular}{|l|l|l|l|l|}
\hline & $\begin{array}{l}\% \text { épargne } \\
\text { liquide }\end{array}$ & $\begin{array}{l}\% \text { actifs } \\
\text { liquides / } \\
\text { actifs totaux }\end{array}$ & $\begin{array}{l}\% \text { ménages recourant au } \\
\text { crédit consommation } \\
\text { cartes de crédit) }\end{array}$ & $\begin{array}{l}\text { Encours moyen } \\
\text { d'emprunt personnel }\end{array}$ \\
\hline $\begin{array}{l}\text { Ménages } \\
\text { hyperboliques }\end{array}$ & $40 \%$ & $39 \%$ & $51 \%$ & $3400 \$$ \\
\hline Ménages exponentiels & $73 \%$ & $50 \%$ & $19 \%$ & $900 \$$ \\
\hline Données réelles PSID & $42 \%$ & $8 \%$ & $70 \%$ & $4600 \$$ \\
\hline
\end{tabular}

L'étude empirique de Laibson, Repeto et Tobacman [2004] estime la structure des taux d'intérêt psychologiques dans le cadre d'un modèle quasi-hyperbolique à l'aide de la méthode des moments simulés. Le calage sur les données réelles de consommation et d'investissement d'une fonction d'actualisation exponentielle fait ressortir un taux d'intérêt psychologique de $15 \%$ par an qui apparaît passablement élevé. Dans ces conditions, la valeur actuelle d'un «util» dans 10 ans n'est que de 0,25 «util». L'étude conclut à un taux psychologique à court terme de $40 \%$ et à long terme de $4,3 \%$. La spécification quasihyperbolique domine empiriquement la spécification d'un taux d'intérêt psychologique constant. Précisons que ces taux sont des estimations en nominal. Paserman [2002] aboutit respectivement à des chiffres de 10 à $60 \%$ pour le taux psychologique à court terme et à $0,1 \%$ pour le taux à long terme. Fang et Siverman [2002] trouvent pour leur part respectivement $57 \%$ et $8 \%$. Gollier et Zeckhauser [2003] obtiennent aussi à partir d'un taux de préférence psychologique constant de 5\% par an en moyenne pour les agents, un taux de préférence social décroissant qui est de $7,5 \%$ à court terme et converge vers $0 \%$ à long terme. Une estimation numérique de la structure des prix psychologiques du temps est proposée pour le consommateur américain par La Bruslerie et Fouilloux [2006] sur des données obligataires. La gamme des taux d'impatience ressort décroissante. Elle est en valeur de l'ordre de 3,5 à $4 \%$ à court terme et converge vers $0 \%$ à moyen-long terme. Ces estimations sont cohérentes avec celles de Hansen et Singleton [1982] pour le court terme et avec les prévisions du modèle hyperbolique.

D'autres études sur données réelles ont été menées en dehors de référence à un modèle théorique spécifique, ce qui conduit le plus souvent à des tests sur données agrégées. Les études réelles présentent l'avantage d'utiliser des données résultant de choix individuels 
effectifs. Par exemple, Feather et Shaw [1999] utilisent la notion de coût d'opportunité du salaire pour estimer le coût d'opportunité des loisirs. Ce dernier est traditionnellement analysé comme une fraction du taux de salaire. La demande de loisirs (ici les loisirs nautiques) dépend tout autant du temps que du prix. La méthode traditionnelle d'estimation du coût d'opportunité du temps bute sur les cas d'individus qui ne sont pas salariés ou qui n'ont pas de salaires observables. De plus, le temps de travail n'est pas discrétionnaire au niveau de l'individu et n'est pas une variable d'ajustement facile à optimiser. Le prix du temps («value of time ») apparaît plus complexe que la simple détermination à partir du taux de salaire et fait intervenir des éléments hédoniques.

L'estimation de la valeur implicite du temps de loisir («shadow price of leisure time ») a aussi été proposée par Lew et Larson [2005] pour tester le salaire discrétionnaire. Cette notion suppose que l'individu est à l'équilibre travail-loisir et peut arbitrer. L'estimation du prix du temps implicite du loisir est stochastique. Elle résulte de manière jointe de l'estimation de la consommation de loisirs sur différentes plages californiennes. Les prix du temps implicites ressortent assez différents. Ils sont plus élevés pour des personnes employés ou suremployés et plus faibles pour des individus à la retraite, étudiants ou chômeurs.

Warner et Pleeter [2001] ont étudié les choix de près de 60000 militaires américains à qui il était proposé de quitter l'armée moyennant une indemnité. Celle-ci était versée soit de manière unique et immédiate, soit sous forme de rente annuelle pendant 18 ans. Le taux actuariel utilisé pour calculer la rente était de 17,5\%, alors qu'à l'époque du choix le taux d'intérêt sur le marché financier était de l'ordre de $7 \%$. Le taux de $17,5 \%$ était financièrement très intéressant et constituait un avantage important en faveur du choix financièrement rationnel de la rente. L'étude révéla que seulement la moitié des officiers et près de $10 \%$ des sous-officiers, employés et soldats choisirent la rente annuelle. Ce résultat empirique illustre une très forte préférence pour le présent. Les taux d'intérêt psychologiques estimés par Warner et Pleeter [2001] furent ainsi entre 24\% et $42 \%$.

Shapiro [2005] a aussi effectué une étude empirique de la fonction de préférence temporelle d'agents américains dans leur comportement de consommation suite à la distribution gratuite de bons de nourriture («food stamps»). Ces subventions à la consommation alimentaire sont distribuées chaque mois. Or, la consommation calorique des ménages bénéficiaires diminue de 10 à $15 \%$ au cours du mois de subvention. Cela indiquerait une préférence pour le court terme relativement plus forte. La calibration d'une fonction de préférence temporelle est effectuée en considérant un investisseur logarithmique $(\alpha=1)$. Shapiro obtient un coefficient d'actualisation journalier de 0.996 , c'est à dire sous l'hypothèse exponentielle un coefficient d'actualisation annuel de 0,23 pour un 1 an. Le taux d'intérêt psychologique, qui en ressort de l'étude de Shapiro [2005], est de 320\% par an. Cette estimation peu raisonnable conduit l'auteur à mettre en cause le modèle d'actualisation exponentiel. Shapiro [2005] calibre alors la fonction d'actualisation quasi-hyperbolique de Laibson [1996]. Il aboutit à un coefficient $\beta$ de 0.96 et conclut que la forme quasihyperbolique correspond mieux aux comportements observés avec néanmoins des taux à court terme très élevés.

\footnotetext{
${ }^{34}$ Le montant de l'indemnité était différent selon le grade (officier/non officier) et le nombre d'années de service.
} 


\subsection{Les études expérimentales}

Les études expérimentales privilégient les choix et les réponses individuelles à des stimuli mettant en jeu leurs préférences. En ce sens elles sont plus riches que les données empiriques agrégées. Le recours à l'économie ou à la psychologie expérimentale est justifié par de nombreux travaux qui visent à mettre en relation des mesures subjectives et des stimuli présents ou passés. Le meilleur argument pour considérer que ces expériences individuelles mesurables comme ayant un sens scientifique est que les fonctions de réaction subjectives bien qu'intimement liées à un individu, sont qualitativement semblables entre ceux-ci (Kahneman et al ., 1997, Broihanne et al., 2004).

Une étude expérimentale de Barsky et al. (1997) ouvre la voie à une mise en cause de l'hypothèse de préférence temporelle. Réalisée sur un petit nombre d'individus, elle montre un possible taux d'actualisation psychologique négatif à partir de la comparaison de profils de consommation future. Une autre étude de Andreoni et Sprenger (2009) ne conclut pas, non plus, à des taux d'actualisation décroissants. Un argument méthodologique évoqué à l'encontre de fonctions d'actualisation décroissante de type hyperbolique est que la mesure de la durée perçue est plus condensée et compacte que la durée objective (Takeuchi, 2008). Si tel est le cas un effet de mesure peut expliquer la forme décroissante des prix t psychologiques du temps (Zauberman et al, 2009) ${ }^{35}$. Quand les réponses sont corrigées de ce bais, l'hypothèse de fonction d'actualisation exponentielle, et donc de taux d'actualisation constants et non décroissants avec le temps, est confirmée.

La théorie des perspectives («prospect theory») (Kahneman et Tversky, 1979 et 1992) est aussi mobilisée dans l'évaluation des prix du temps, pour appréhender la manière dont les agents réagissent différemment aux perspectives présentes ou futures. Les individus décident en fonction de motivations intrinsèques mais ils forment aussi des anticipations déformées à partir des croyances probabilistes objectives. Cette conception met en évidence l'existence de nombreux biais cognitifs par lesquels les individus sont influencés au travers de schémas mentaux, des raccourcis heuristiques, par une rationalité qui les conduit à adopter des comportements adaptatifs. La préférence temporelle en elle-même semble neutre par rapport aux concepts d'adaptation (encore appelé « ancrage ») qui se manifestent avec un aléa sur un résultat. L'aversion asymétrique au risque affecte aussi l'aléa de résultats de l'expérimentation.

Les expérimentations menées en économie et finance comportementale ont été principalement centrées sur la problématique de l'aversion au risque (algorithme d'ancrage et asymétrie des gains et des pertes). Il est classiquement demandé à un agent de se prononcer envers des loteries de gains monétaires (Boihanne et al., 2004). S'il existe de nombreux travaux expérimentaux sur le prix psychologique du temps, il en existe peu sur la structure à terme des taux d'escompte psychologiques. Khwaja et al. [2006] montrent sur un panel d'individu que les préférences temporelles subjectives sont différentes et qu'elles relèveraient de l'inné. Les études du taux marginal de préférence temporelle de Kurz et al. [1973] fondée sur un questionnaire simple aboutissent à des prix psychologique de $36 \%$ à $76 \%$ par an. Dans leur étude Thaler et Shefrin [1981], utilisent une question simple: "quel bonus d'argent demanderiez-vous aujourd'hui plutôt que de recevoir un bonus de 100 dollars dans un an?». Les variables influençant le taux de préférence temporelle sont classiquement l'âge, le niveau

\footnotetext{
${ }^{35}$ Ces auteurs font référence sur ce point à Pigou et à la loi de Weber-Fechner, qui introduit une relation décroissante entre un stimulus et sa perception.
} 
de revenu et le statut marital. Thaler et Shefrin [1981] soulignent le rôle de l'âge : les jeunes doivent encore apprendre (et internaliser) les techniques de self-control. Une structure des prix psychologique du temps décroissante est largement évoquée dans la littérature. Quand deux revenus sont anticipés dans le long terme par rapport à aujourd'hui, les agents sont relativement patients. Le coût de l'attente marginal est alors faible (par exemple, selon Thaler et Shefrin [1981], «je préfère 2 pommes dans 101 jours qu'une pomme dans 100 jours »). Quand les deux revenus sont proches, l'impatience est plus grande : "je préfère une pomme tout de suite plutôt que deux pommes demain ».

Frederick et al. [2002] reprennent les estimations moyennes des facteurs d'actualisation d'issues d'une trentaine d'études expérimentales. Ils associent à ces facteurs d'actualisation l'horizon des choix proposés qui va du très court terme (10 dollars aujourd'hui contre $\mathrm{X}$ dollars demain) jusqu' au long terme (10 dollars aujourd'hui contre Y dollars dans 10 ans). Frederick, Loewenstein et Donoghue [2002] discutent cependant les termes de comparaison dans le temps qui sont des revenus monétaires. A juste titre ils se méfient d'une pollution des réponses par une proximité ou une confusion avec le taux d'intérêt.

Figure 1 - Estimation expérimentale du prix psychologique du temps
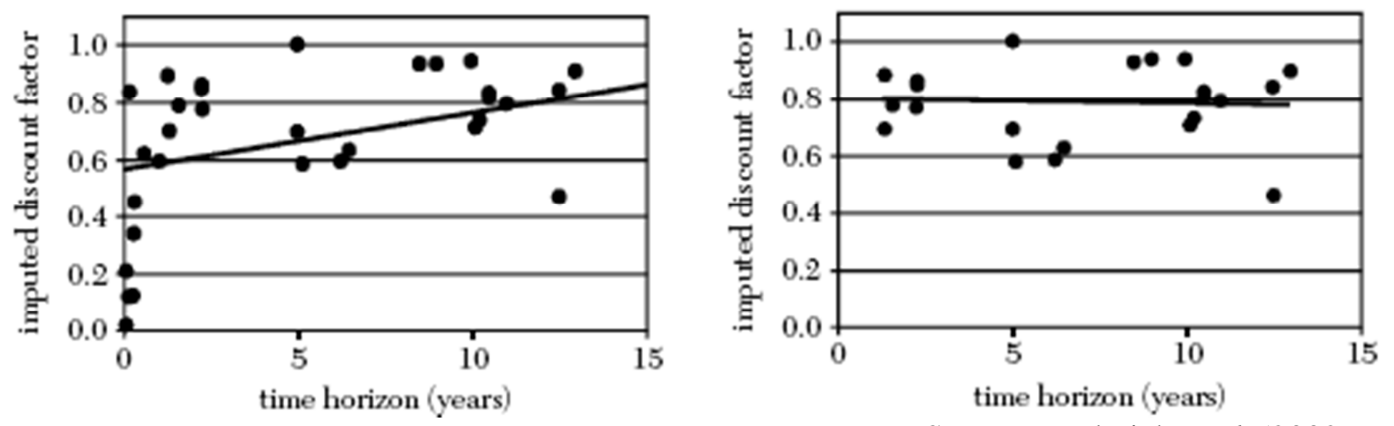

Source: Frederick et al. (2002, p. 362)

La figure 1 met en évidence l'ensemble des estimations expérimentales en fonction de l'horizon temporel d'estimation. La régression montre une pente croissante des coefficients d'actualisation $\delta(t)$ et donc une structure décroissante des taux d'escompte psychologique. La partie droite de la figure 1 ne présente que les estimations expérimentales de choix intertemporels au-delà de l'horizon de 1 an, c'est-à-dire excluant le court terme. Les points sont alignés et la droite de régression a une pente quasi-nulle. Ces résultats suggèrent que la pente des taux psychologique est très décroissante à court terme, puis plate par la suite. L'existence d'une structure par terme des prix psychologiques du temps a des conséquences importantes dans l'analyse des choix individuels intertemporels. La rationalité intertemporelle des choix individuels devient plus complexe et la relation standard entre utilité et décision est mise en cause.

Une question importante que suggèrent les études expérimentales concernant les protocoles ou les questionnaires relatifs à des attitudes face au temps est de savoir si les choix doivent être présentés en terme monétaires ou non (Soman et al., 2005). L'expression des préférences temporelles exprimées en en termes monétaires, c'est à dire en pouvoir d'achat, a tendance conduire à une sous-estimation de la valeur des réponses exprimées. En effet, la présentation des alternatives avec l'épargne et le taux d'intérêts est source de confusion dans la formulation des réponses par les individus. Comparer des sommes entre maintenant et demain renvoie à un choix d'épargne implicite difficile à formuler. Comparer des sommes 
monétaires enter le présent et l'avenir est un choix d'épargne. Cette décision est perturbée la possibilité de différer du pouvoir d'achat en utilisant un actif atteignable sur un marché financier, ici le taux d'intérêt. Quand les choix sont monétaires, un comportement normatif et rationnel s'impose en suivant la comparaison introduite par Fisher entre le taux de substitution marginal des deux flux monétaires présent et futur avec le taux d'intérêt. Read [2004] fait remarquer que «discount rates that exceed the opportunity cost of capital represent irrational behavior ». La préférence temporelle est une catégorie pure, indépendante du contenu de choix présents et futurs, qui portent sur des biens différents. Epargne et consommation sont des biens différents. Il semble alors totalement préférable de comparer des utilités liées à des consommations d'un bien donné entre maintenant et la consommation du même bien dans le futur. Cairns et Van der Poole [2000, 2002] ont ainsi d'abord procédé à des enquêtes en numéraire avant de privilégier des études fondées sur le fait de retarder dans le temps une maladie. Une évolution méthodologique semblable est mise en évidence dans l'étude empirique de Barsky, Juster, Kimball et Shapiro [1997], où les profils de consommation sont dénommés en dollarsvet le test de Shapiro [2005], où des bons de nourriture exprimés en calories sont utilisés. L'utilisation de «time-tradeoff » (TTO) est une méthodologie nouvelle, qui ne requiert aucune hypothèse sur les fonctions d'utilité (Rhode, 2010). Une étude expérimentale sur un échantillon, menée par Attema et al. [2009], montre que les fonctions d'actualisation seraient de formes plus diverses que celle attendue par l'hypothèse d'impatience. Ces auteurs retiennent même l'idée d'impatience croissante, c'est-à-dire de taux d'actualisation croissants avec l'horizon pour certains individus. Signalons aussi l'apparition récente d'analyses neurophysiologiques, qui ouvrent aussi une voie de recherche sur la compréhension des choix intertemporels avec des protocoles spécifiques (Kalenscher et. Pennartz, 2008).

\subsection{La mesure individuelle du prix psychologique du temps}

Les études sur la mesure individuelle du prix psychologique du temps procèdent du même protocole expérimental en s'interrogeant sur la perception d'un gain plus petit maintenant ou d'un gain plus important plus tard. La plupart de ces études supposent une fonction d'utilité linéaire sur des biens de consommation et considèrent aussi qu'il n'existe pas de fongibilité intertemporelle (c'est à dire que le gain est supposé être consommé au moment où il est reçu). Ces études - surtout en psychologie - ont identifié les liens entre le prix psychologique du temps et les caractéristiques de l'individu. Un grand nombre de ces études ont concerné des populations particulières d'individus : les utilisateurs de drogue, les joueurs, les individus impulsifs, les individus atteints de troubles psychiatriques (Reynolds, 2006 pour une présentation générale). D'autres études analysent le lien entre le prix psychologique du temps et les traits de l'individu, tel l'âge ou les aptitudes cognitives).

Les études de la mesure individuelle du prix psychologique du temps identifient les déterminants suivants :

- Les fumeurs: La majorité des expériences montre une relation entre l'usage de tabac et la fonction d'actualisation, établissant que les fumeurs sont caractérisés par un prix psychologique du temps plus important (Backer et al., 2003, Kirby et Perty, 2004, Ohmura et al., 2005).

- Les alcooliques : Les études font apparaître que les alcooliques ont prix psychologique du temps plus élevé. A titre d'exemple, les alcooliques sérieux actualisent davantage les gains que les alcooliques abstinents (Petry, 2001a). Les alcooliques-dépendants 
désintoxés ont aussi un prix psychologique du temps plus important que les alcooliques contrôlés (Bjork et al., 2004).

- Les utilisateurs de drogues illicites: Selon des études récentes (Bretteville-Jensen, 1999, Kirby et Petry, 2004), il existe une relation positive entre le prix psychologique du temps et les usages de drogues illicites principalement la cocaïne, l'héroïne et les amphétamines.

○ Les joueurs : Les joueurs pathologiques ont aussi un prix psychologique du temps plus élevés que la population. Petry [2001b] montre notamment que les joueurs avec une grande fréquence de jeux au cours des trois mois précédent l'expérience ont taux d'actualisation très élevé.

- L'âge: Les expériences appliquées montrent la patience augmente avec l'âge, les jeunes accordant ayant un prix psychologique du temps très faible en comparaison avec une population plus âgée (Green et al., 1994). Toutefois, Read et Read [2004] mettent en évidence que les adultes les plus âgés (en moyenne 75 ans) représentent le groupe de population le plus patient lorsque les délais de temps sont inférieurs à 1 an. Cependant, cette étude montre aussi que les plus âgés sont aussi le groupe de population le moins patient lorsqu'il s'agit de délais de 3 à 10 ans. Ces résultats laissent apparaître que les individus les plus âgés sont particulièrement sensibles à un risque de mortalité à l'horizon de 3 à 10 ans.

- Les aptitudes cognitives : elles ont un rôle singulièrement souligné par Frederick [2005]. Il utilise dans un test expérimental un indice de réflexion cognitive (CRT «Cognitive reflection test») qui oppose des individus qui formulent des réponses intuitives sans réfléchir à ceux qui font des choix ou prennent des décisions réfléchies. Cet indice est croisé sur un échantillon de 3428 répondants avec deux dimensions importantes des choix : la préférence temporelle et l'attitude envers le risque (aversion ou attrait). Le premier résultat est de monter que l'indice de réflexion cognitive est lié à la patience : plus les individus sont réfléchis et ont une capacité d'analyse plus ils sont alors patients. Le lien entre le CRT et l'attitude envers le risque est aussi établi. Les individus avec un fort CRT sont plus tolérants au risque ${ }^{36}$.

- Le genre est aussi un trait fondamental dans l'évaluation psychologique du temps. Frederick [2005] a montré que le niveau de CRT dépend du genre. Les femmes ont un CRT plus bas 1,01 que celui des hommes 1,47. Le score CRT est ici très corrélé avec la préférence temporelle pour les femmes.

- L'aversion au risque semble aussi exercer une influence. Pour Frederick [2005], il existe un facteur commun derrière la préférence pour le temps et le risque. «Les préférences pour le temps et le risque sont parfois liées si fortement dans la mesure des capacités cognitives qu'ils en constituent en eux-mêmes une mesure directe » ${ }^{37}$. Cette piste intéressante conduit à se poser la question de savoir si les deux dimensions de l'aversion au risque et de la structure de préférence temporelle seraient liées.

Les résultats des études empiriques en psychologie comportementale laissent donc apparaitre que la perception de l'horizon temporel est fortement dépendante des caractéristiques

\footnotetext{
${ }^{36}$ L'asymétrie entre gains et pertes est confirmés au sens de la théorie des perspectives de Kahneman et Tversky [1979]. L'aversion au risque des individus avec un CRT bas est plus forte pour éviter les pertes et sont prêt à parier dans ce but alors qu'ils ne parient pas lorsqu'il s'agit de gains et préfèrent les gains certain.

${ }^{37}$ Cf. art. cit., p.39
} 
individuelles des agents. Ces propositions confortent ainsi l'idée qu'il est nécessaire de considérer une valeur psychologique du temps spécifique pour l'évaluation temporelle de l'utilité ressentie individu par individu.

\section{Conclusion}

L'analyse de la littérature sur le prix psychologique du temps met en lumière la grande diversité des approches théoriques, renouvelées par les apports des tests expérimentaux. Le schéma rationnel de l'utilité escomptée à un taux d'escompte subjectif constant apparaît comme très limitatif. Les choix décisionnels de l'agent dans le temps résultent de motifs individuels et psychologiques conditionnant le caractère intertemporel des choix. La détermination du prix psychologique du temps appelle ainsi à une reconsidération de la temporalité des choix de l'individu ainsi que du rapport entre le «moi » présent et le(s) «moi(s)» futur(s). Le modèle standard de l'utilité escomptée a été ainsi profondément remis en cause par les études sur la forme de l'actualisation hyperbolique. Se pose immédiatement alors la question de la rationalité des choix intertemporels et l'ouverture d'une forme de la rationalité limitée compatible avec une incohérence temporelle des choix. L'intégration des éléments de continuité et de différentiation des «mois» successifs conduit aussi à reconsidérer le caractère stable des choix intertemporels de l'agent. La distinction entre la notion d'utilité de la décision calculée (qui découle des choix) et celle d'utilité ressentie ou simplement reçue (qui fait appel à l'introspection et à l'appréhension rétrospective des choix concernant des plaisirs et des peines passées ou qui est reçue sans découler directement de choix) permet d'élargir la problématique et de se départir de la stricte cohérence rationnelle des choix. Divers travaux empiriques ont souligné les divergences dans la représentation temporelle du calcul économique des agents (par exemple dans l'accumulation patrimoniale, les choix publics, environnement....) selon la forme du prix psychologique du temps considéré.

Les recherches sur la fonction d'actualisation psychologique sont loin d'être épuisées. Une question reste pendante sur l'association croisée entre l'attitude face au risque et l'attitude face au temps. Un programme majeur restant à défricher est celui de la compréhension des antécédents psychologiques et neurophysiologiques de la préférence temporelle et des changements de forme des fonctions d'utilité. Cette étude de la littérature sert de point de départ à la conduite d'une réflexion sur l'existence et surtout d'une structure temporelle de la valeur psychologique du temps. Le recours à des expériences comportementales dont la problématique consisterait ainsi étudier les fondements de la valeur subjective du temps dans le temps s'avère nécessaire. Cette extension de la représentation standard nécessite notamment de prendre en compte les caractéristiques individuelles des agents et plus particulièrement d'intégrer la possibilité de révision des choix en fonction de leurs probabilités de survie. 


\section{Références bibliographiques}

AKERLOF G. [1991] : "Procrastination and obedience", American Economic Review, vol.81 $\mathrm{n}^{\circ} 2$, pp. 1-19

Ainslie G. [1992] : Picoeconomics, Cambridge, Cambridge University Press.

Ainslie G., HASlam N. [1992] : «Hyperbolic Discounting », In George Loewenstein and John Elster (eds.), Choice over time, New York: Russell Sage Foundation, pp. 57-92.

Andreoni J. Sprenger C. [2009] : «Estimating time preference from convex budget », Working paper UCSD.

Angeletos G.M., D. Laibson, A. Repetto, J. Tobacman et S. Weinberg [2001] : «The hyperbolic buffer stock model : calibration, simulation and empirical evaluation », Journal of Economic Perspectives, vol.15, pp.47-68.

Arrondel L, Masson A. Verger [2004] : «Mesurer les préférences individuelles pour le présent », Economies et Statistiques, n³7, pp 87-125.

Attema A., Han Bleichrodt, Kirsten Rohdeb, Peter. Wakker [2009] : « Time tradeoff sequences for analyzing discounting and time inconsistency », Working paper Erasmus University.

BARSKy R., Juster F., Kimball M. et Shapiro M. [1997] : «Preference parameters and behavioral heterogeneity: An experimental approach in the health and retirement study », Quarterly Journal of Economics, vol.112, pp. 537-579.

Bentham J. [1789] : An Introduction into the Principles of Morals and Legislation, New York, Hafner Library

BJork J.M., Hommer DW., GRANT S.J. et DANUBE C. [2004] : «Impulsivity in abstinent alcohol-dependent patients: relation to control subjects and type 1-/type 2- liketraits », Alchohol, vol.34, pp. 133-150.

BREEDEN D. [1986] : «Consumption, production, inflation and interest rates », Journal of Financial Economics, vol.16, pp. 3-29.

BRetTEVILle-Jensen A.L. [1999] : «Addiction and discounting », Journal of Health Economics, vol.18, pp. 393-407.

Broihanne M-H., M. Merli et P. Roger [2004] : Finance comportementale, Collection Gestion, Economica.

Buchanan [1969] : Cost and choice, Markham Publishing co, Chicago.

CAIRns J., VAN DER POOL M. [2000] : «Valuing future private and social benefits: the discounted utility model versus hyperbolic discounting », Journal of Economic Psychology, vol. 21, pp. 191-205.

CAIRnS J., VAN DER POOL M. [2002] : «A comparison of the discounted utility model and hyperbolic discounting models in the case of social and private intertemporal preferences for health », Journal of Economic Behavior and Organization, vol. 49, pp. 79-96. 
CAMPBell J. et J. Cochrane [1999] : «By force of habit: A consumption-based explanation of aggregate stock market behaviour », Journal of Political Economy, vol.107, pp. 205-251.

Chung S. et J. HerRnstein [1961] : «Relative and absolute strengths of response as a function of frequency of reinforcement », Journal of Experimental analysis of animal behaviour, vol.4, pp. 267-272.

Cocco J., Gomes F. et P. Maenhout [2005] : « Consumption and portfolio choice over life cycle », Review of Financial Studies, vol.18(2), pp.491-533.

EPSTEIN L. et S. ZIN [1989] : "Substitution, risk aversion and the temporal behavior of consumption and asset returns : A theoretical framework », Econometrica, vol.57(4), pp. 937969.

EPSTEIN L. et S. ZIN [1991] : «Substitution, risk aversion and the temporal behavior of consumption and asset returns : An empirical analysis », Journal of Political Economy, vol. 99(2), pp. 263-288.

FANG H. et D. SILVERMAN [2002] : «Time-inconsistency and welfare program participation : Evidence from NLSY », Avril 2002, working paper.

FEATHER P., SHAW W. [1999]: «Estimating the cost of leisure for recreation demand models », Journal of Environmental Economics and Management, vol.38, pp. 49-65

FISHER I., [1930] : The theory of interest, New York, MacMillan.

FREDERICK S. [2005] : « Cognitive Reflection and Decision Making », Journal of Economic Perspectives, Vol; 19, Number 4, Fall 2005, pp. 24-42.

FREDERICK S. [2006] : «Valuing future life and futures lives: A framework for understanding discounting », Journal of Economic Psychology, vol. 27, pp. 667-680.

Frederick S., Loewenstein G. et T. O’Donoghue [2002] : «Time discounting and time preference: A critical review », Journal of Economic Litterature, vol. 40(1), pp. 351-401.

Green L., Fry A.F. et Myerson J. [1994] : «Discounting of delayed rewards: A life-span comparison », Psychological Science, vol. 5, pp. 33-37.

GOLLIER C. [2002] : «Time horizon and the discount rate », Journal of Economic Theory, vol. 107 , pp. $463-473$.

GOllier C. et R. ZeCKHAUSER [2005] : «Collective investment decision making with heterogeneous times preferences », Journal of Public Economics, CESifo, working paper $n^{\circ} 915$, ssrn.com.

HANSEN L., SingletON K. [1982] : «Generalized instrumental variables estimation of non linear rational expectations models », Econometrica, vol. 50, pp. 1269-1286.

HANSEN L., Singleton K. [1983] : "Stochastic consumption, risk aversion and the temporal behaviour of asset returns », Journal of Political Economy, vol. 91, pp. 249-268.

HARRIS C., LAIBSON D. [2001] : «Hyperbolic discounting and consumption », working paper, février 2001, Harvard University.

HARRIS C., LAIBSON D. [2001] : «Dynamic choices of hyperbolic consumers », Econometrica, vol .69, n 4, pp. 935-957. 
HARRIS C., LAIBSON D. [2004] : «Intantaneous gratification », Working paper, Harvard University.

HAYEK F. [1973-1979] : Edition française [1982], Droit, législation et liberté, PUF, Paris.

JOUINI E. et C. NAPP [2010] : «Cognitive biases and the representative agent », working paper, halshs-00488570, version 1.

Tobias Kalenscher , Cyriel M.A. Pennartz [2008] : « Is a bird in the hand worth two in the future? The neuroeconomics of intertemporal decision-making », Progress in Neurobiology, vol. 84, pp. 284-315.

KAHNEMAN D. [1994]: «New challenges to the rationality assumption », Journal of Institutional and Theoretical Economics, vol. 150, pp. 18-36.

Kahneman D. et A. TVersky [1979] : «Prospect Theory: An Analysis of Decision under Risk », Econometrica, Vol. XVLII, pp. 263-291.

KAHNEMAN D. et RIIS J. [2005] : «Living, and thinking about it: Two perspectives on life »,. In F.A. Huppert, N. Baylis \& B. Keverne (Eds.), The science of well-being (pp. 285-304). Oxford: Oxford University Press.

KAhNeman D., WAKKer P. et SARIN R. [1997] : «Back to Bentham? Exploration of experienced utility », Quarterly Journal of Economics, vol. 112, pp.375-405

Khwaja A., F. SLOAN et M. SALm [2006] : vEvidence on preference and subjective beliefs of risk takers: The case of smokers », International Journal of Industrial Organization, vol. 24, pp. 667-682.

KIRBY K.N. et N.M. PETRY [2004] : « Heroin and cocaine abusers have higher discount rates for delayed rewards than alcoholics or non-drug-using controls », Addiction, Vol. 99, pp. 461471.

KoOPMANS T [1960] : «Stationary ordinal utility and impatience », Econometrica, Vol. 28 $\mathrm{n}^{\circ} 2$, pp. 287-309.

LA BRUSLERIE de H. et J. FouillouX [2006]: «La structure par terme des prix psychologiques du temps: Une modélisation décroissante », Working paper, PRISMSorbonne, Université Paris 1.

LAIBSON D. [1996] : «Hyperbolic discount functions, undersaving and saving policy », WP $\mathrm{n}^{\circ} 5635, \mathrm{NBER}$

LAIBSON D. [1997] : «Golden eggs and hyperbolic discounting », Journal of Political Economy, vol. 112, pp. 443-477.

Laibson D., A. RepetTo et J. Tobacman [2004] : «Estimating Discount functions from lifecycle consuption choices », working paper, Harvard University.

LELOUP S. [2002] : «Conséquences anticipées et comportements face au risque dans la pensée économique de Jeremy Bentham », Revue Economique, vol. 53(3), pp. 415-423.

LENGWILER, Y. [2005] : « Heterogeneous patience and the term structure of real interest rates », American Economic Review, Vol. 95, pp. 890-896; 
LOEWENSTeIn G., PReleC D. [1992] : «Anomalies in intertemporal choice : Evidence and an interpretation », Quarterly Journal of Economics, vol. 57, pp. 573-598.

MAzuR, J. E. [1987] : «An Adjusting Procedure for Studying Delayed Reinforcement », In Michael L. Commons, James E. Mazur, John A. Nevin, \& Howard Rachlin (eds.), Quantitative Analyses of Behavior 5, 55-73, Lawrence Erlbaum, Hillsdale NJ

MAZUR, J. E. [2001] : «Hyperbolic value addition and general models of animal choice. », Psychological Review, Vol. 108, pp. 96-112.

Mehra R. et Prescot E. [1985] : «The equity premium : A puzzle », Journal of Monetary Economics, vol. 15, pp. 145-161.

NocetTI, D., Jouini, E., C. NAPP [2008] « Properties of the social discount rate in a Benthamite framework with heterogeneous degrees of impatience », Management Science, Vol. 54, pp. 1822-1826.

O’Donoghue T. et M. Rabin [1999] : « Doing it now or later », American Economic Review, vol.89(1), pp. 103-124.

OHMURA Y., TAKAHAShi T. et N. KitAmura [2005]: " "Discounting delayed and probabilistic monetary gains and losses by smokers of cigarettes », Psychopharmacology, vol. 182, pp. 508-515.

PASERMAN D. [2002] : «Job search and hyperbolic discounting: structural estimation and policy evaluation », working paper, Hebrew University.

PETRY N. [2001] : " Delay discounting of money and alcohol in actively using alcoholics, current abstinent alcoholics, and controls », Psychopharmacology, vol. 154, pp. 243-250.

PRELEC, DRAZEN [2004] : «Decreasing Impatience: A Criterion for Non-Stationary Time Preference and "Hyperbolic" Discounting », Scandinavian Journal of Economics, Vol. 106, pp. 511-532.

RAE J. [1832] : "Statement of some principles on the subject of political economy"

RAMSEY J. [1928] : «A mathematical theory of saving », Economic Journal, December, pp. 543-559.

RAWLS J. [1971] : A theory of justice, Cambridge MA, Harvard Press University.

READ D. et NL READ [2004] : «Time discounting over the lifespan », Organization Behaviour and Human Decision Processes, vol.94, pp. 22-32.

ReAD D., S. Frederick, B. ORSEL, J. RAHMAN [2005] : «Four Score and Seven Years from Now: The Date/Delay Effect in Temporal Discounting », Management Science, Vol. 51 (September), p. 1326-35.

REYNOLDS B. [2006] : «A review of dealy-discounting research humans : relations to drug use and gambling », Behavioural Pharmacology, vol. 17, 651-667.

RoHDE K. [2010] : «The hyperbolic factor: A measure of time inconsistency », Journal of Risk and Uncertainty, vol. 41, pp. 125-140. 
SAMUElson, P. [1937] : «A note on measurement of utility », Review. Economics Studies vol. 4, pp. 155-161.

SCHMiDT C. [2006] : «Psychologie cognitive et analyse des décisions économiques », Revue Française d'Economie, vol.20 n³, pp.3-51

Soman D., Ainslie G.,Li Xiuping, Lynch J., Mitchell A., Read D., Sawyer A., Trope Y., Wertenbroch K., Zauberman G. [2005] : « The Psychology of Intertemporal Discounting: Why are Distant Events Valued Differently from Proximal Ones? », Marketing Letters, 16, 3/4, pp.347-360

SHAPIRO J. [2005] : «Is there a daily discount rate? Evidence from the food stamp nutrition cycle », Journal of Public Economics, vol. 89, pp. 303-325.

STROTZ R. [1956] : «Myopia and inconsistency in dynamic utility maximisation », Review of Economic studies, vol. 23, pp. 165-180.

TAKEUCHI, KAN (2008) : «When does the Future really start? Nonmonotonic Time Preference », Working Paper.

THALER R. [1981] : «Some empirical evidence on dynamic inconsistency », Economic Letters, vol. 8, pp. 201-207.

THALER R., SHEFRIN H. [1981] : «An economic theory of self-control », Journal of political Economy, vol. 89 n², pp. 392-406.

TVERSKY A. et D. KAHNEMAN [1992] : «Advances in prospect theory: Cumulative representation of uncertainty », Journal of Risk and Uncertainty, vol. 5, pp. 297-323.

Von Mises L. [1949]: Human Action, a Treatise on Economics, New Haven: Yale University Press.

WARner J., Pleeter S. [2001] : «The personal discount rate: Evidence from military downsizing programs, American Economic Review, vol. 91, pp. 33-53.

ZAUBERMAN G. (2003] : «The intertemporal dynamics of Consumer-Lock-In », Journal of Consulmer Research, vol.30, dec, pp405-419.

Zauberman G., Kim K., Malkoc S., Bettman J., [2009], «Discounting Time and Time Discounting:Subjective Time Perception and Intertemporal Preferences », Journal of Marketing Research, Vol. XLVI, pp. 543-556. 